\title{
Optimal blockwise subcarrier allocation policies in single-carrier FDMA uplink systems
}

\author{
Antonia Maria Masucci ${ }^{1} 2^{*}$, Elena Veronica Belmega ${ }^{1}$ and Inbar Fijalkow ${ }^{1}$
}

\begin{abstract}
In this paper, we analyze the optimal (blockwise) subcarrier allocation schemes in single-carrier frequency division multiple access (SC-FDMA) uplink systems without channel state information at the transmitter side. The presence of the discrete Fourier transform (DFT) in SC-FDMA/orthogonal frequency division multiple access OFDMA systems induces correlation between subcarriers which degrades the transmission performance, and thus, only some of the possible subcarrier allocation schemes achieve better performance. We propose as a performance metric a novel sum-correlation metric which is shown to exhibit interesting properties and a close link with the outage probability. We provide the set of optimal block-sizes achieving the maximum diversity and minimizing the inter-carrier sum-correlation function. We derive the analytical closed-form expression of the largest optimal block-size as a function of the system's parameters: number of subcarriers, number of users, and the cyclic prefix length. The minimum value of sum-correlation depends only on the number of subcarriers, number of users and on the variance of the channel impulse response. Moreover, we observe numerically a close strong connection between the proposed metric and diversity: the optimal block-size is also optimal in terms of outage probability. Also, when the considered system undergoes carrier frequency offset (CFO), we observe the robustness of the proposed blockwise allocation policy to the CFO effects. Numerical Monte Carlo simulations which validate our analysis are illustrated.
\end{abstract}

Keywords: SC-FDMA/OFDMA; Subcarriers allocation; Channel frequency diversity; Cyclic prefix induced; Correlation; Carrier frequency offsets

\section{Introduction}

Due to its simplicity and flexibility to subcarrier allocation policies, single-carrier frequency division multiple access (SC-FDMA) has been proposed as the uplink transmission scheme for wireless standard of $4 \mathrm{G}$ technology such as 3GPP long-term evolution (LTE) [1-3]. SC-FDMA is a technique with similar performance and essentially the same general structure as an orthogonal frequency division multiple access (OFDMA) system. A remarkable advantage of SC-FDMA over OFDMA is that the signal has lower peak-to-average power ratio (PAPR) that guarantees the transmit power efficiency at the mobile terminal level [4]. However, similarly to OFDMA, SC-FDMA shows sensitivity to small values of carrier frequency offsets (CFOs) generated by the frequency misalignment

\footnotetext{
*Correspondence: antonia.masucci@inria.fr

1 ETIS/ENSEA - University of Cergy Pontoise - CNRS, 6 Avenue de Ponceau,

95014 Cergy, France

${ }^{2}$ INRIA Paris-Rocquencourt, Le Chesnay Cedex, France
}

between the mobile users' oscillators and the base station [5-7]. CFO is responsible for the loss of orthogonality among subcarriers by producing a shift of the received signals causing inter-carrier interferences (ICI).

In this work, we show that the SC-FDMA uplink systems without $\mathrm{CFO}$ and with imposed independent subcarriers attain the same channel diversity gain for any subcarrier allocation scheme. However, due to the discrete Fourier transform (DFT) of the channel, correlation between the subcarriers is induced, and thus, a degradation of the transmission performance occurs. Therefore, there exist some allocation schemes that are able to achieve an increased diversity gain when choosing the appropriate subcarrier allocation block-size.

In the uplink SC-FDMA transmissions, users spread their information across the set of available subcarriers. Subcarrier allocation techniques are used to split the available bandwidth between the users. In the case in which no channel state information (CSI) is available at the transmitter side, the most popular allocation scheme is the

\section{量 Springer}

(c) 2014 Masucci et al: licensee Springer. This is an Open Access article distributed under the terms of the Creative Commons Attribution License (http://creativecommons.org/licenses/by/4.0), which permits unrestricted use, distribution, and reproduction in any medium, provided the original work is properly credited. 
blockwise allocation in [8]. In this blockwise allocation scheme, subsets of adjacent subcarriers, called blocks, are allocated to each user, (see Figures 1, 2, and 3). In particular, we call mono-block allocation the scheme with the maximum block-size given by the ratio between the number of subcarriers and the number of users, illustrated in Figure 1 . The interleaved allocation scheme is a special case in which subcarriers are uniformly spaced at a distance equal to the number of users (block-size $b$ is equal to one), as shown in Figure 3. The interleaved allocation is usually considered to benefit from frequency diversity (IEEE 802.16) [9]. However, robustness to CFO can be improved by choosing large block-sizes since they better combat the ICI. In the case of full CSI, an optimal blocksize has been proposed for OFDMA systems in [10] as a good balance between the frequency diversity gain and robustness against CFO. In this paper, we study the optimal block-size allocation schemes, in the case of an uplink SC-FDMA without CSI.

To the best of our knowledge, the closest works to ours are references [11,12]. A subcarrier allocation scheme with respect to the user's outage probability has been proposed in [11] for OFDMA/SC-FDMA systems with and without CFO. In particular, the authors of [11] propose a semi-interleaved subcarrier allocation scheme capable of achieving the diversity gain with minimum CFO interference. However, the authors analyze only the case in which every user in the system transmits one symbol spread to all subcarriers, and as a consequence, their diversity results are restricted to the considered model and with a low data rate. We point out that our main contributions with respect to [11] consist in the following: we consider a more general model; we analyze all possible subcarrier allocation block-sizes; and we find the analytical expressions of the optimal blockwise allocation schemes that achieve maximum diversity. Moreover, we provide an analytical expression of the correlation between subcarriers and we analyze its effects on the system transmission's performance.

More precisely, in this work, we propose a new allocation policy based on the minimization of the correlation between subcarriers. In particular, in order to optimize the block-size subcarrier allocation, we propose a new performance metric, i.e., the sum-correlation function that we define as the sum of correlations of each subcarrier with respect to the others in the same allocation scheme. The introduction of the sum-correlation function as a performance metric is motivated by the fact that the correlation generated by the DFT implies that some allocation schemes achieve a higher diversity gain than others. The interest of the proposed approach is due to the fact that it allows us to find the exact expression of the block-sizes that achieve a higher diversity gain. It turns out that the minimum sum-correlation is achieved by block-size allocation policies that lie in a set composed of all block-sizes that are inferior or equal to a given threshold depending explicitly on the system's parameters: the number of subcarriers, the number of users, and the cyclic prefix length. Furthermore, we find the minimum value of the sumcorrelation function. This value guarantees to achieve the maximum diversity gain, and what is more remarkable, it depends only on the number of subcarriers, number of users, and the variance of the channel impulse response. We also provide interesting properties of the individual sum-correlation terms: the auto-correlation term (i.e., the correlation between the subcarrier of reference and itself) depends on the length of cyclic prefix; the correlations between the subcarrier of reference and the ones that are spaced from it of a distance equal to a multiple of the ratio between the number of subcarriers, and the cyclic prefixes are equal to zero. The most interesting property of the proposed sum-correlation function is the close link to the outage probability and thus to the diversity gain. Numerically, we observe that the maximum diversity or the minimum outage allocation coincides with the one minimizing our sum-correlation function.

Moreover, we observe that when the SC-FDMA system undergoes $\mathrm{CFO}$, we have the robustness to $\mathrm{CFO}$ for practical values of CFO. This means that when the CFO goes to zero, the CFO sum-correlation can be approximated with the sum-correlation defined in the case without CFO. This analysis has been done similarly to [12] in which coded

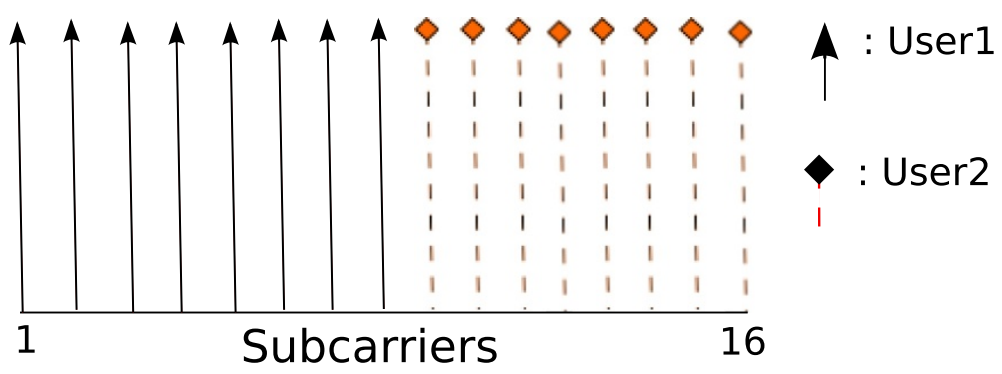

Figure 1 Mono-block allocation scheme. Block-size $b=8, N_{p}=16$ subcarriers, and $N_{u}=2$ users (subcarriers allocated to user $u_{1}$ denoted by arrow markers and to user $u_{2}$ by diamond markers). 


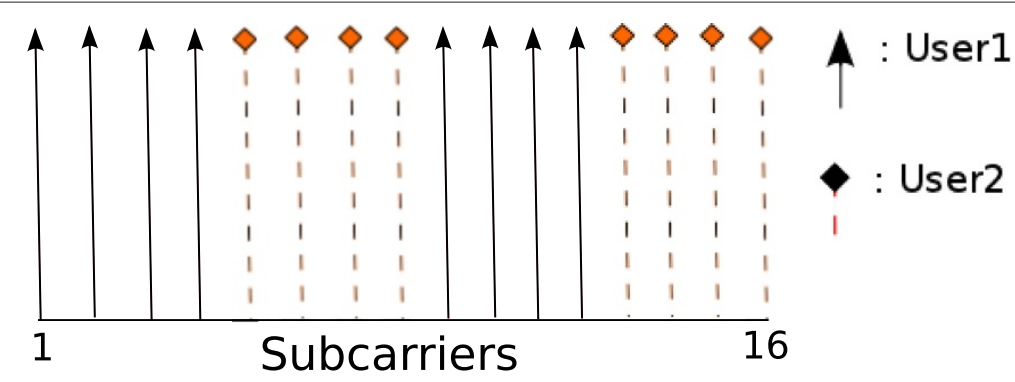

Figure 2 Blockwise allocation scheme. Block-size $b=4, N_{p}=16$ subcarriers, $N_{u}=2$ users (subcarriers allocated to user $u_{1}$ denoted by arrow markers and to user $u_{2}$ by diamond markers).

OFDMA systems are analyzed. Uncoded OFDMA cannot exploit the frequency diversity of the channel; therefore, the use of channel coding with OFDMA in [12] reduces the errors resulting from the multipath fading environment recovering the diversity gain. Coding is not needed in SC-FDMA since it can be interpreted as a linearly precoded OFDMA system [4].

We underline that, with respect to [12] in which the results have been briefly announced, in this paper: 1) we provide a deeper and a more detailed theoretical analysis; 2) we consider a new performance metric, not identical to the one analyzed in [12], which takes into account the length of the channel impulse response $L_{h} \leq L$ and a general power delay profile which allow us to generalize our previous results in both cases, with and without CFO; 3 ) novel simulation results are presented in order to validate these new results.

The difficulty of our analytical study is related to the discrete feasible set of allocation block-sizes and also to the objective function (i.e., the sum-correlation function we propose) which is closely linked with the outage probability whose minimization is still an open issue in most non-trivial cases [13]. However, we provide extensive numerical Monte Carlo simulations that validate our analysis and all of our claims.

The sequel of our paper is organized as follows. In Section 2, we present the analytical model of the SCFDMA system without CFO. In Section 3, we define a novel sum-correlation function and its properties; moreover, we find the optimal block-sizes for a subcarrier allocation scheme minimizing the subcarrier correlation function and we show the numerical results that validate our analysis. We present the SC-FDMA system with CFO in Section 4. We define the corresponding sum-correlation function and we observe its robustness against CFO. Numerical results that validate this analysis are also presented. At last, in Section 5 we conclude the paper.

\section{System model without CFO}

We consider a SC-FDMA uplink system where $N_{u}$ mobile users communicate with a base station (BS) or access point. In the case in which the system is not affected by CFOs, the users are synchronized to the BS in time and frequency domains. No CSI is available at the transmitter side. The total bandwidth $B$ is divided into $N_{p}$ subcarriers and we denote by $M=\left\lfloor\frac{N_{p}}{N_{u}}\right\rfloor$ (where $\lfloor x\rfloor$ is the integer part of $\mathrm{x}$ ) the number of subcarriers per user. Notice that we choose $N_{p}$ as an integer power of two in order to optimize the DFT processing. To provide a fair allocation of the spectrum among the users (fair in the sense that the number of allocated subcarriers is the same for all users), notice that the number of not-allocated carriers is $N_{p}-N_{u} M<N_{u}<<N_{p}$ which is a negligible fraction of the total available spectrum. Without loss of

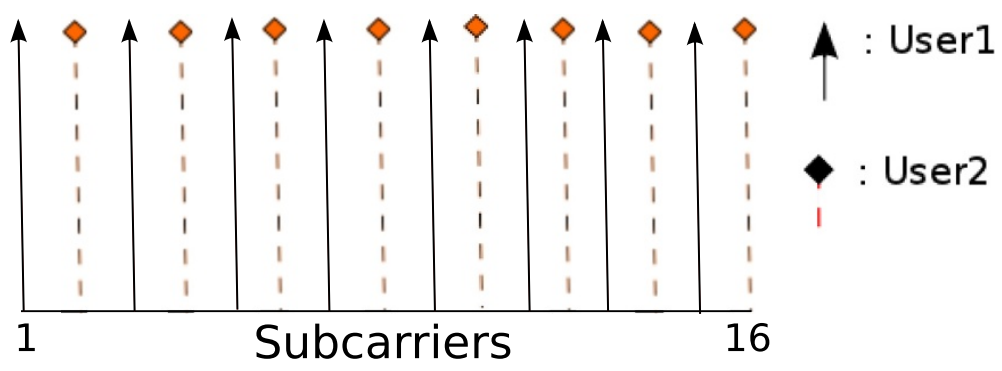

Figure 3 Interleaved allocation scheme. Block-size $b=1, N_{p}=16$ subcarriers, $N_{u}=2$ users (subcarriers allocated to user $u_{1}$ denoted by arrow markers and to user $u_{2}$ by diamond markers). 
generality and also to avoid complex notations ${ }^{\mathrm{a}}$, we will assume in the following that $N_{u}$ is also a power of two and that $M=\frac{N_{p}}{N_{u}}$.

The signal at the input of the receiver DFT was expressed in [10] as follows:

$$
\begin{aligned}
& \left(\begin{array}{c}
y_{N_{p}-1} \\
\vdots \\
y_{0} \\
\vdots \\
y_{-L}
\end{array}\right)=\sum_{u=1}^{N_{u}}\left(\begin{array}{ccccc}
h_{0}^{(u)} & \cdots & h_{L_{h}-1}^{(u)} & & \\
& & & & 0 \\
& \ddots & & \ddots & \\
0 & & & & \\
& & h_{0}^{(u)} & \cdots & h_{L_{h}-1}^{(u)}
\end{array}\right) \\
& \times\left(\begin{array}{c}
a_{N_{p}-1}^{(u)} \\
\vdots \\
a_{0}^{(u)} \\
\vdots \\
a_{-L}^{(u)}
\end{array}\right)+\left(\begin{array}{c}
n_{N_{p}-1} \\
\vdots \\
n_{0} \\
\vdots \\
n_{-L}
\end{array}\right)
\end{aligned}
$$

where $L$ is the length of the cyclic prefix. The vector $\mathbf{h}^{(u)}=$ $\left[h_{0}^{(u)}, \ldots, h_{L_{h}-1}^{(u)}\right]$ is the channel impulse response whose dimension $L_{h}$ is lower than or equal to $L$. The elements $a_{k}^{(u)}$ are the symbols at the output of the inverse discrete Fourier transform (IDTF) given by

$$
\mathbf{a}^{(u)}=\left(\begin{array}{c}
a_{N_{p}-1}^{(u)} \\
\vdots \\
a_{0}^{(u)}
\end{array}\right)=\mathbf{F}^{-1} \boldsymbol{\Pi}_{b}^{(u)} \mathbf{x}^{(u)}
$$

with $\mathbf{F}^{-1}$ the $N_{p}$-size inverse DFT matrix, $\mathbf{x}^{(u)}=\mathbf{F}_{\frac{N_{P}}{N_{u}}} \tilde{\mathbf{x}}^{(u)}$ where $\mathbf{F}_{\frac{N_{P}}{N_{u}}}$ is the $\frac{N_{p}}{N_{u}}$-size DFT matrix, and $\tilde{\mathbf{x}}^{(u)}$ is the vector of the $M$-ary symbols transmitted by user $u$. The vector $\tilde{\mathbf{x}}^{(u)}$ does not have a particular structure, contrary to [11] where it is assumed to be equal to $\mathbf{1}_{\frac{N_{p}}{N_{u}} \times 1} \tilde{x}$ which means that one symbol is spread to all subcarriers. The symbol $\Pi_{b}^{(u)}$ is the $N_{p} \times \frac{N_{p}}{N_{u}}$ subcarrier allocation matrix with only one element equal to 1 in each column which occurs at rows that represent the carriers allocated to user $u$ according to the considered block-size $b \in \beta=$ $\left\{1, \ldots, \frac{N_{p}}{N_{u}}\right\}$. The set $\beta$ is composed of all divisors of $\frac{N_{p}}{N_{u}}$, this guarantees a fully utilized spectrum. The SC-FDMA can be viewed as a pre-coded version of OFDMA since the $\frac{N_{p}}{N_{u}}$-size DFT matrix does not affect the channel diversity. Discarding in the signal at the input of the receiver DFT the $L$ components corresponding to the cyclic prefix and rearranging the terms, we get

$$
\begin{aligned}
\underbrace{\left(\begin{array}{c}
y_{N_{p}-1} \\
\vdots \\
y_{0}
\end{array}\right)}_{\mathbf{y}}=\sum_{u=1}^{N_{u}}\left(\begin{array}{ccccc}
h_{0}^{(u)} & \cdots & h_{L_{h}-1}^{(u)} & & 0 \\
& \ddots & & \ddots & \\
& & & & h_{L_{h}-1}^{(u)} \\
h_{L_{h}-1}^{(u)} & 0 & & & \\
\vdots & \ddots & & \ddots & \\
h_{1}^{(u)} & \cdots & h_{L_{h}-1}^{(u)} & & h_{0}^{(u)}
\end{array}\right) \\
\times\left(\begin{array}{c}
a_{N_{p}-1}^{(u)} \\
\vdots \\
a_{0}^{(u)}
\end{array}\right)+\left(\begin{array}{c}
n_{N_{p}-1} \\
\vdots \\
n_{0}
\end{array}\right)
\end{aligned}
$$

where $\mathbf{h}_{\text {circ }}^{(u)}$ is a $N_{p} \times N_{p}$ circulant matrix. Denoting $\mathbf{r}=\mathbf{F y}$, we have found that the received signal at the BS after the $N_{p}$-size DFT is given by:

$$
\begin{aligned}
\mathbf{r} & =\sum_{u=1}^{N_{u}} \mathbf{F h}_{\mathrm{circ}}^{(u)} \mathbf{F}^{-1} \boldsymbol{\Pi}_{b}^{(u)} \mathbf{x}^{(u)}+\mathbf{F} \mathbf{n} \\
& =\sum_{u=1}^{N_{u}} \mathbf{H}^{(u)} \boldsymbol{\Pi}_{b}^{(u)} \mathbf{x}^{(u)}+\tilde{\mathbf{n}}
\end{aligned}
$$

where $\mathbf{H}^{(u)}=\mathbf{F} \mathbf{h}_{\text {circ }}^{(u)} \mathbf{F}^{-1}$ is the diagonal channel matrix of user $u$ with the diagonal $(k, k)$-entry given by

$$
H_{k}^{(u)}=\frac{1}{\sqrt{N_{p}}} \sum_{m=0}^{L_{h}-1} h_{m}^{(u)} e^{-j 2 \pi m k / N_{p}},
$$

and $\tilde{\mathbf{n}}=$ Fn is the $N_{p} \times 1$ additive Gaussian noise with variance $\sigma_{n}^{2} \mathbf{I}$. Therefore, over each subcarrier $k=0, \ldots, N_{p}-$ 1 , we have

$$
r_{k}=\sum_{u=1}^{N_{u}} \frac{1}{\sqrt{N_{p}}} \sum_{m=0}^{L_{h}-1} h_{m}^{(u)} e^{-j 2 \pi m k / N_{p}} \Pi_{k, k}^{(u)} x_{k}^{(u)}+n_{k} .
$$

Note then that $\mathbf{H}^{(u)}$ is diagonal thanks to the assumption on the channel impulse response length being shorter than the cyclic prefix [10]. However, the diagonal entries (5) are correlated with each other.

\section{Minimization of the subcarriers sum-correlation} Frequency diversity occurs in OFDMA systems by sending multiple replicas of the transmitted signal at different carrier frequencies. The idea behind diversity is to provide independent replicas of the same transmitted signal at the receiver and appropriately process them to make the detection more reliable. Different copies of the signal should be transmitted in different frequency bands, a condition which guarantees their independence. The subchannels given in (5) are correlated and no more than a $L_{h}$-order frequency diversity gain can be possible since there are only $L_{h}$ independent channel coefficients, i.e., 
$h_{0}^{(u)}, \ldots, h_{L_{h}-1}^{(u)}$, [14]. Intuitively, we can imagine that there are $L_{h}$ groups of $\frac{N_{p}}{L_{h}}$ frequencies which are 'identical'. Each user retrieves the maximal diversity if it has at least $L_{h}$ blocks of size $b$ which implies $b \leq \frac{N_{p}}{L_{h} N_{u}}$. Therefore, users can achieve full diversity when the block-size is within the coherent bandwidth $\frac{N_{p}}{L_{h} N_{u}}$.

In this work, we propose an original approach to find the block-size that guarantees the maximum diversity. This approach is based on the minimization of the subchannels/subcarrier sum-correlation function.

In the sequel, we define a measure of correlation between subcarriers, that we call sum-correlation function, and we derive its properties. Moreover, we find the set of optimal block-sizes $b^{*} \in \beta$ which minimizes this correlation in order to minimize the effects that it produces.

\subsection{Properties of the sum-correlation function}

Assuming that the channel impulse responses are independent but distributed accordingly to the complex Gaussian distribution $\mathcal{C N}\left(0, \sigma_{h}^{(u) 2}\right)$ [14], we define for each user the sum of correlations of each subcarrier with respect to the others in the same allocation scheme as follows:

$$
\begin{aligned}
\Gamma_{u, m}(b)= & \sum_{c_{u} \in \mathcal{C}_{u}} \mathbb{E}\left[H_{m}^{(u)} H_{c_{u}}^{(u) *}\right] \\
= & \mathbb{E}\left[\left|H_{m}^{(u)}\right|^{2}\right]+\sum_{\substack{c_{u} \in \mathcal{C}_{u} \\
c_{u} \neq m}} \mathbb{E}\left[H_{m}^{(u)} H_{c_{u}}^{(u) *}\right] \\
= & \frac{\sigma_{h}^{(u) 2}}{N_{p}} L_{h}+\frac{\sigma_{h}^{(u) 2}}{N_{p}} \sum_{c_{u} \in \mathcal{C}_{u}} \frac{\sin \left[\frac{\pi L_{h}}{N_{p}}\left(m-c_{u}\right)\right]}{\sin \left[\frac{\pi}{N_{p}}\left(m-c_{u}\right)\right]} \\
& \times e^{-\pi j \frac{\left(L_{h}-1\right)}{N_{p}}\left(m-c_{u}\right)}
\end{aligned}
$$

where $m \in \mathcal{C}_{u}$ is the reference subcarrier and

$$
\mathcal{C}_{u}=\bigcup_{k \in\left\{1,2,3, \ldots, \frac{N_{p}}{b N_{u}}\right\}}\left\{(k-1) b N_{u}+(u-1) b+i, \forall i \in\{1, \ldots, b\}\right\} .
$$

The set $\mathcal{C}_{u}$ is composed of all indices of subcarriers allocated to user $u$ given a block-size $b$ allocation scheme.
The ratio $\frac{N_{p}}{b N_{u}}$ represents the number of blocks that can be allocated to each user given a block-size $b$. We consider, therefore, that the total $N_{p}$ subcarriers are divided into $\frac{N_{p}}{b N_{u}}$ large-blocks that contain $b N_{u}$ subcarriers corresponding to the $N_{u}$ blocks, one for each user, of size $b$. The set $\mathcal{C}_{u}$ is the union of indices of subcarriers allocated to user $u$ in all these large-blocks. Inside of the large-block of index $k$, the indices of the $b$ subcarriers allocated to user $u$ are $(k-1) b N_{u}+(u-1) b+i, \forall i \in\{1, \ldots, b\}$, where $(k-1) b N_{u}$ corresponds to the previous $k-1$ large-blocks and $(u-1) b$ corresponds to the previous allocated users $(1,2, \ldots, u-1)$, see Figure 4 . The function $\Gamma_{u, m}(b)$ is the sum of the correlations between subcarriers that are in the same allocation scheme.

Considering the subcarriers $m$ and $c_{u}$ in $\mathcal{C}_{u}$, we denote the distance between them by

$$
\begin{aligned}
d & :=m-c_{u} \\
& =\left(k^{\prime}-k^{\prime \prime}\right) b N_{u}+i^{\prime}-i^{\prime \prime}
\end{aligned}
$$

with $k^{\prime}, k^{\prime \prime} \in\left\{1,2,3, \ldots, \frac{N_{p}}{b N_{u}}\right\}$ and $i^{\prime}, i^{\prime \prime} \in\{1, \ldots, b\}$. We define the function

$$
f(d) \triangleq \begin{cases}L_{h} & \text { if } d=0 \\ \frac{\sin \left[\pi \frac{L_{h}}{N_{p}} d\right]}{\sin \left[\frac{\pi}{N_{p}} d\right]} e^{-\pi j \frac{\left(L_{h}-1\right)}{N_{p}} d} & \text { otherwise }\end{cases}
$$

The next result guarantees that the function $f(d)$ is independent of the user index.

Proposition 1. Given the parameters $N_{p}, N_{u}$, and $L_{h}$, the value $f(d)$ of the function in (9) for any $d=m-c_{u}$ in (8) is independent of the user index $u$.

Proof: The dependence of $f$ on the user index $u$ is expressed by the term $\left(m-c_{u}\right)$, representing the distance between two subcarriers in the same allocation scheme, where $m, c_{u} \in \mathcal{C}_{u}$. If $m$ and $c_{u}$ are in two different blocks there exist two indices $k^{\prime}$ and $k^{\prime \prime}$ in $\left\{1,2,3, \ldots, \frac{N_{p}}{b N_{u}}\right\}$ such that

$$
\begin{aligned}
& m=\left(k^{\prime}-1\right) b N_{u}+(u-1) b+i^{\prime} \\
& c_{u}=\left(k^{\prime \prime}-1\right) b N_{u}+(u-1) b+i^{\prime \prime}
\end{aligned}
$$

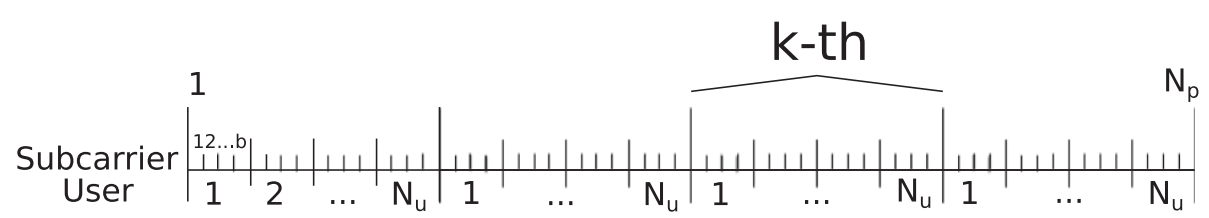

Figure 4 Set of subcarriers. The total $\boldsymbol{N}_{\boldsymbol{p}}$ subcarriers are divided into $\frac{\boldsymbol{N}_{\boldsymbol{p}}}{\boldsymbol{b N}_{\boldsymbol{u}}}$ large blocks. The $k$ th large block contains $b N_{u}$ subcarriers, which are divided into $N_{u}$ block of size $b$, one for each user. 
with $i^{\prime}$ and $i^{\prime \prime}$ in $\{1, \ldots, b\}$. Therefore, the distance

$$
m-c_{u}=\left(k^{\prime}-k^{\prime \prime}\right) b N_{u}+i^{\prime}-i^{\prime \prime}
$$

does not depend on the user index $u$. If $m$ and $c_{u}$ are in the same block, $k^{\prime}=k^{\prime \prime}$ and the same reasoning holds. This guarantees the independence of the function $f(d)$ on the particular user $u$.

The independence between $f(d)$ and user index $u$ comes from the fact that given a block-size $b$, the set of distances between subcarriers are the same for all users.

We observe that the function $f(d)$ has the following circularity property.

Proposition 2 (Circularity of $\mathrm{f}(\mathrm{d})$ ). Given $N_{p}$ the number of subcarriers and for any distance $d$ between subcarriers given in (8), we have

$$
f\left(d+N_{p}\right)=f(d) .
$$

Proof: From (9),

$$
\begin{aligned}
f\left(d+N_{p}\right) & =\frac{\sin \left[\pi \frac{L_{h}}{N_{p}}\left(d+N_{p}\right)\right]}{\sin \left[\frac{\pi}{N_{p}}\left(d+N_{p}\right)\right]} e^{-\pi j \frac{\left(L_{h}-1\right)}{N_{p}}\left(d+N_{p}\right)} \\
& =\frac{e^{j \pi \frac{L_{h}}{N_{p}} d} e^{j \pi L_{h}}-e^{-j \pi \frac{L_{h}}{N_{p}} d} e^{-j \pi L_{h}}}{e^{j \pi \frac{1}{N_{p}} d} e^{j \pi}-e^{-j \pi \frac{1}{N_{p}} d} e^{-j \pi}} \frac{L_{h} d}{N_{p} d \pi \frac{1}{N_{p}} d} e^{-j \pi} \\
& =\frac{1-e^{-j 2 \pi \frac{L_{h}}{N_{p}} d} e^{-j 2 \pi L_{h}}}{1-e^{-j 2 \pi \frac{1}{N_{p}} d} e^{-j 2 \pi}} \\
& =\frac{e^{j \pi \frac{L_{h}}{N_{p}} d}-e^{-j \pi \frac{L_{h}}{N_{p}} d}}{e^{j \pi \frac{1}{N_{p}} d}-e^{-j \pi \frac{1}{N_{p}} d} e^{-j \pi \frac{\left(L_{h}-1\right)}{N_{p}} d}} \\
& =\frac{\sin \left[\pi \frac{L_{h}}{N_{p}} d\right]}{\sin \left[\frac{\pi}{N_{p}} d\right]} e^{-\pi j \frac{\left(L_{h}-1\right)}{N_{p}} d} \\
& =f(d) .
\end{aligned}
$$

We consider the following sum-correlation metric:

$$
\Gamma(b)=\sum_{u=1}^{N_{u}} \sum_{m \in \mathcal{C}_{u}} \Gamma_{u, m}(b) .
$$

Thanks to Proposition 1 and Proposition 2, it can be expressed as follows:

$$
\begin{aligned}
\Gamma(b) & =\sum_{u=1}^{N_{u}}\left|\mathcal{C}_{u}\right| \frac{\sigma_{h}^{(u) 2}}{N_{p}}\left(\sum_{d \in \mathcal{D}_{b}} f(d)\right) \\
& =\sum_{u=1}^{N_{u}} \frac{N_{p}}{N_{u}} \frac{\sigma_{h}^{(u) 2}}{N_{p}}\left(L_{h}+\sum_{\substack{d \in \mathcal{D}_{b} \\
d \neq 0}} f(d)\right)
\end{aligned}
$$

where

$$
\mathcal{D}_{b}=\left\{\begin{array}{l}
d=k b N_{u}+i, i \in\{0, \ldots, b-1\} \\
k \in\left\{0, \ldots,\left(\frac{N_{p}}{b N_{u}}-1\right)\right\}
\end{array}\right\} .
$$

Given a subcarrier of reference, without loss of generality, the set $\mathcal{D}_{b}$ represents the set of the distances ${ }^{\mathrm{b}}$ between the subcarrier of reference and all the other subcarriers in the same allocation scheme (the $k$ factor represents here the distance between the large-blocks). This definition is consistent since the function $f(d)$ has the circularity property with respect to $N_{p}$. This means that it does not matter which subcarrier of reference we consider. Therefore, the sum-correlation function defined in (15) is independent on the reference subcarrier. This guarantees that the next results hold for each user in the system.

We observe that there is no correlation between the subcarrier of reference and other carriers which are spaced from it at a distance equal to a multiple of $\frac{N_{p}}{L_{h}}$. It is obvious from the definition of the function $f(d)$ that it is equal to zero when the distance $d$ is a multiple of the ratio $\frac{N_{p}}{L_{h}}$ :

$$
f\left(r \frac{N_{p}}{L_{h}}\right)=0, \quad \forall r \in \mathbb{N}^{*} .
$$

This is what we observe in Figure 5, in which we plot the absolute value of the function $|f(d)|$, with $m=1, N_{p}=$ $32, N_{u}=2$ and $L_{h}=4$. We observe that this function is equal to zero for all the multiples of $\frac{N_{p}}{L_{h}}=8$.

We have seen that the function $f(d)$ equals zero for all multiples of $\frac{N_{p}}{L_{h}}$ and that the distance $d$ can be expressed in function of the block-size $b$ (see (8) and (17)). In the following, we provide the expression of the block-size $b$ such that $d=\frac{N_{p}}{L_{h}}$.

Proposition 3. Given a fixed distance $d=\frac{N_{p}}{L_{h}}$ between subcarriers, the corresponding block-size is equal to $b=$ $\frac{N_{p}}{L_{h} N_{u}}$.

Proof: Notice that, given our allocation policy in Figure 4, not all the distances can be achieved for any possible block-size. We consider an arbitrary distance $d$ in $\mathcal{D}_{b}$ 


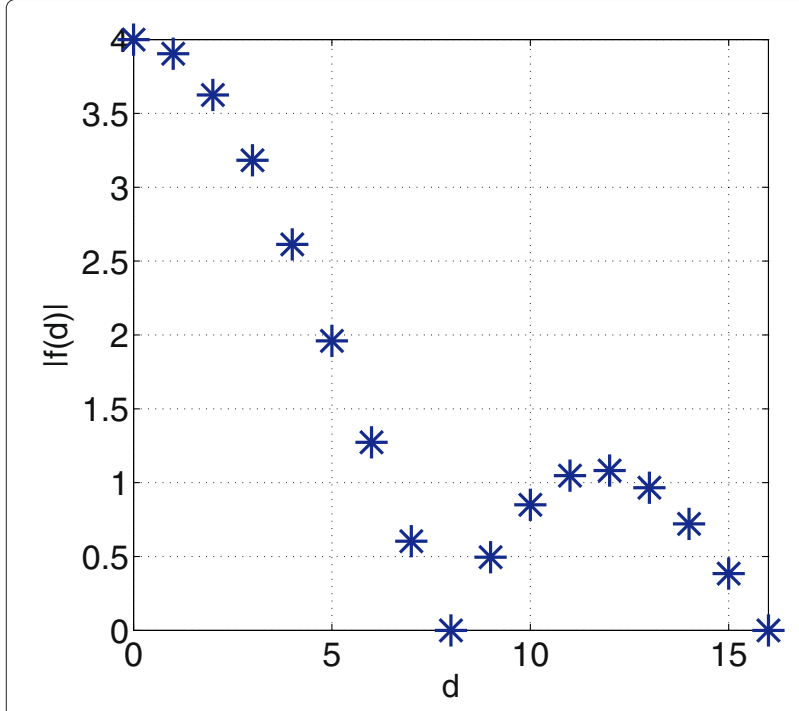

Figure 5 The function $|f(d)|$, with $m=1, N_{p}=32, N_{u}=2$, and $\boldsymbol{L}_{\boldsymbol{h}}=\mathbf{4}$. In this case, $\frac{N_{p}}{L_{h}}=8$.

and we are interested to find the block-size that ensures $d=\frac{N_{p}}{L_{h}}$ :

$$
\begin{aligned}
d & =k b N_{u}+i \text { with } i \in\{0, \ldots, b-1\}, \\
& \quad \text { and } k \in\left\{0, \ldots, \frac{N_{p}}{b N_{u}}-1\right\} \\
& =r \frac{N_{p}}{L_{h}} \quad \text { with } r \leq 1 .
\end{aligned}
$$

First, we analyze the case when $b \geq \frac{N_{p}}{L_{h}}$. In this case, we observe that the condition $d=\frac{N_{p}}{L_{h}}$ is satisfied if $k=0$ and $i=\frac{N_{p}}{L_{h}}$. This means that for all $b \geq \frac{N_{p}}{L_{h}}$ there are two subcarriers in the same block such that their distance is $\frac{N_{p}}{L_{h}}$. More interesting is the case when $b<\frac{N_{p}}{L_{h}}$. From the fact that $i \in\{0, \ldots, b-1\}$ and $b<\frac{N_{p}}{L_{h}}$, in order to have the distance $d=\frac{N_{p}}{L_{h}}$, we have just to consider the case $i=0$. Then, it is obvious that we have $d$ equal to $\frac{N_{p}}{L_{h}}$ when $b N_{u}=\frac{N_{p}}{L_{h}}$. This means that the block-size is $b=\frac{N_{p}}{L_{h} N_{u}}$.

\subsection{Novel blockwise allocation scheme}

In the next Theorem, we give the set of optimal block-sizes that minimize the sum-correlation function $\Gamma(b)$. This set is given by $\beta^{*}=\left\{1, \ldots, \frac{N_{p}}{N_{u} L}, \frac{N_{p}}{N_{u} L_{h}}\right\} \subseteq \beta$.

Theorem 1. We consider our uplink system with $N_{p}$ subcarriers, $N_{u}$ users and a channel impulse response length $L_{h}$. Given $\beta^{*} \triangleq\left\{1, \ldots, \frac{N_{p}}{N_{u} L}, \frac{N_{p}}{N_{u} L_{h}}\right\}$, we have
1. The elements in the set $\beta^{*}$ minimize the sum-correlation function $\Gamma(b)$ :

$$
\beta^{*}=\underset{b \in \beta}{\arg \min } \Gamma(b)
$$

2. The optimal value of the sum-correlation function depends only on the system parameters.

$$
\Gamma\left(b^{*}\right)=\sum_{u=1}^{N_{u}} \sigma_{h}^{(u) 2} \frac{N_{p}}{N_{u}^{2}}, \quad \forall b^{*} \in \beta^{*} .
$$

Proof: The proof is given in the Appendix 5.

Proposition 4. In the case without CSI, assuming that $L_{h}$ is not known at the transmitter side, we propose to use $\beta^{*}$ restricted to $\left\{1, \ldots, \frac{N_{p}}{N_{u} L}\right\}$.

Proof: We observe that $\left\{1, \ldots, \frac{N_{p}}{N_{u} L}\right\}$ is included in $\left\{1, \ldots, \frac{N_{p}}{N_{u} L}, \ldots, \frac{N_{p}}{N_{u} L_{h}}\right\}$. Intuitively, this means that, in a more realistic scenario in which only the knowledge of $L$ and not of the channel length $L_{h}$ is available, we can still provide the subset of optimal block-sizes.

We observe that, in the case without CSI, the minimum value of the sum-correlation function depends only on the number of subcarriers, number of users, and the variance of the channel impulse response and that the largest optimal block-size is given by

$$
b_{\max }^{*}=\frac{N_{p}}{N_{u} L}
$$

which is a function of system's parameters: number of subcarriers, number of users, and cyclic prefix length.

In a more general scenario in which the channel length is different for each user, i.e., $L_{h}^{(u)} \neq L_{h}$, the optimal block-size maximizing the sum-correlation function is a difficult problem and an open issue. However, in a realistic scenario in which these parameters $L_{h}^{(u)}$ are not known, the system planner would assume the worse case scenario and approximate them with the length of the cyclic prefix $L$. Since the length of the cyclic prefix $L$ is bigger, the chosen block-length is suboptimal and given by $(21)$.

\subsection{Numerical results: diversity and sum-correlation}

In this section, the aim is to highlight the close relationship between diversity gain, outage probability and the sum-correlation function. We define the outage 
probability of the system under consideration as the maximum of the outage probabilities of the users:

$$
P_{\text {out }}=\max _{1 \leq u \leq N_{u}} P_{\text {out }}^{(u)}
$$

where $P_{\text {out }}^{(u)}=\operatorname{Pr}\left\{C_{b}^{(u)}<R\right\}$ with $R$ is a fixed target transmission rate and $C_{b}^{(u)}$ is the instantaneous mutual information of the user $u$ defined in the following. We consider the transmitted symbols in (2) distributed accordingly to the Gaussian distribution such that $\mathbb{E}\left[\mathbf{x}^{(u)} \mathbf{x}^{(u) H}\right]=\mathbf{I}$. The user instantaneous achievable spectral efficiency assuming single-user decoding at the BS [15] in the case without $\mathrm{CFO}$ is as follows:

$$
\begin{aligned}
C_{b}^{(u)}= & \frac{N_{u}}{B} \log _{2} \operatorname{det}\left[\mathbf{I}+\mathbf{H}^{(u)} \boldsymbol{\Pi}_{b}^{(u)} \mathbf{H}^{(u) \dagger}\right. \\
& \left.\times\left(\mathbf{I} \sigma_{n}^{2}+\sum_{\substack{v=1 \\
v \neq u}}^{N_{u}} \mathbf{H}^{(v)} \boldsymbol{\Pi}_{b}^{(v)} \mathbf{H}^{(v) \dagger}\right)^{-1}\right] \\
= & \frac{N_{u}}{B} \sum_{m \in \mathcal{C}_{u}} \log _{2}\left(1+\frac{1}{\sigma_{n}^{2}}\left|H_{m}^{(u)}\right|^{2}\right) .
\end{aligned}
$$

An explicit analytical relation between the sumcorrelation function and the outage probability is still an open problem. The major issue is the fact that the distribution of the mutual information is very complex and closed-form expressions for the outage probability are not available in general. For example, Emre Telatar's conjecture on the optimal covariance matrix minimizing the outage probability in the single-user MIMO channels [13] is yet to be proven. We propose a new metric, the sumcorrelation function, and show by simulations that there is an underlying relation between the sum-correlation function and the outage probability. Indeed, it is intuitive that, in SC-FDMA systems, correlation among the subcarriers decreases the diversity gain and, thus, the transmission reliability decreases $[16,17]$. This explains that the outage probability increases when the correlation among subcarriers is increasing. This connection has been validated via extensive numerical simulations. The interest behind this connection is that the sum-correlation function has a closed-form expression allowing us to perform a rigorous analysis and to find the blockwise subcarrier allocation minimizing the sum-correlation which is consistent with the optimal blockwise subcarrier allocation minimizing the outage probability. The following results illustrate numerically this connection.

\subsubsection{Uncorrelated subcarriers}

We consider the case of a SC-FDMA system with independent subcarriers. Since the subcarriers are independent the correlation between them is zero, which means that the sum-correlation $\Gamma(b)$ is equal to zero for any blocksize $b$. In the next simulation, we observe that we obtain the same performance in terms of the outage probability regardless of the particular allocation scheme and the block-size, see Figure 6. Although this scenario is unrealistic from a practical standpoint, it is important to notice that, in this case, there are no privileged block-sizes to achieve better diversity gain.

In Figure 6, we plot the outage probability in the SC-FDMA system with independent subcarriers (subchannels) generated by complex Gaussian distribution with respect to SNR for the scenario $N_{p}=64, N_{u}=2$, and fixed rate $R=1 \mathrm{bits} / \mathrm{s} / \mathrm{Hz}$. In particular, in this case with independent subcarriers, we consider the matrix $\mathbf{H}^{(u)}$ in (4) to be diagonal with entries $H_{k}^{(u)}$ i.i.d $\sim \mathcal{C N}\left(0, \sigma^{2}\right)$. It is clear that for any block-size (hence, for any subcarrier allocation scheme) we obtain the same performance in terms of outage probability. Therefore, there are not any privileged block-size allocations to achieve better diversity gain. This motivates and strengthens our observation that the subcarrier correlation has a direct impact on the outage probability.

\subsubsection{Correlated subcarriers}

In this section, we consider a more interesting and realistic SC-FDMA system given in (4). For simplicity and

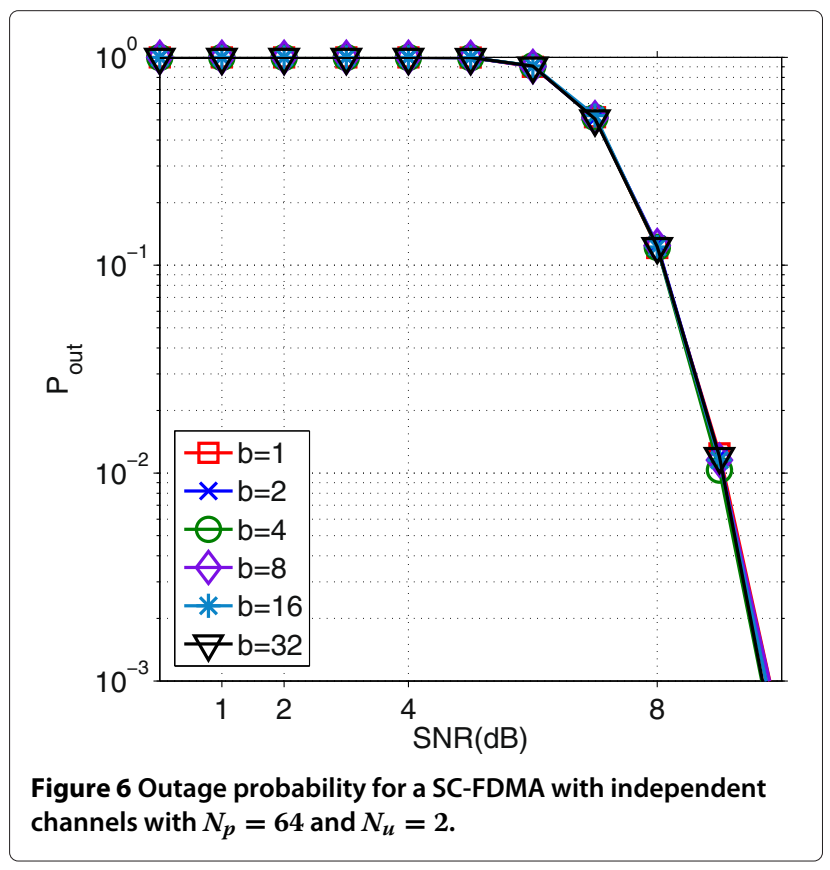


lack of space-related reasons, the simulations presented here have been done for the particular case $L=L_{h}$. Numerous other simulations were performed in the general case $L_{h} \leq L$, which confirm the theoretical result of Theorem 1.

In Figure 7, we have plotted with respect to the blocksize $b$, the sum-correlation function $\Gamma(b)$ in the SC-FDMA system without CFO for the scenario $N_{u}=4, L=4$ and $\sigma^{2}(1)=0.25, \sigma^{2}(2)=0.5, \sigma^{2}(3)=0.125, \sigma^{2}(4)=0.3$. The illustrated markers represent the values of the function $\Gamma(b)$ for the given choice of the parameters of the system. We observe that the minimal values of $\Gamma(b)$ are obtained for the block-sizes $b^{*} \in \beta^{*}=\{1,2,4\}$. In particular, $\forall b^{*} \in \beta^{*}=\{1,2,4\}$ we have $\Gamma\left(b^{*}\right)=\sum_{u=1}^{N_{u}} \frac{\sigma_{h}^{(u) 2} N_{p}}{N_{u}^{2}}=$ 4.7.

In Figure 8, we use Binary Phase Shift Keying (BPSK) modulation in the following scenario: $N_{p}=64, N_{u}=2$, $L=8$, and $\sigma_{h}^{(1) 2}=\sigma_{h}^{(2) 2}$. We observe that the optimal block-sizes are in $\beta^{*}=\{1,2,4\}$ (but here, we just plot the smallest and the biggest values) for the BER which confirms that these block-sizes optimize also the sum-correlation function we have proposed.

In Figure 9, we use BPSK modulation, $N_{p}=64, N_{u}=2$, $L=4$, and $\sigma_{h}^{(2) 2}=2 \sigma_{h}^{(1) 2}$. The optimal block-sizes are given in the set $\beta^{*}=\{1,2,4,8\}$.

In Figure 10, we use BPSK modulation in the following scenario: $N_{p}=128, N_{u}=2$, and $L=8$. In this case, we consider an exponential power delay profile which means that $\sigma_{h}^{(u) 2}=\frac{e^{-\tau / L}}{\sum_{\tau=0}^{L-1} e^{-\tau / L}}$ with $\tau \in\{0,1, \ldots, L-1\}$.

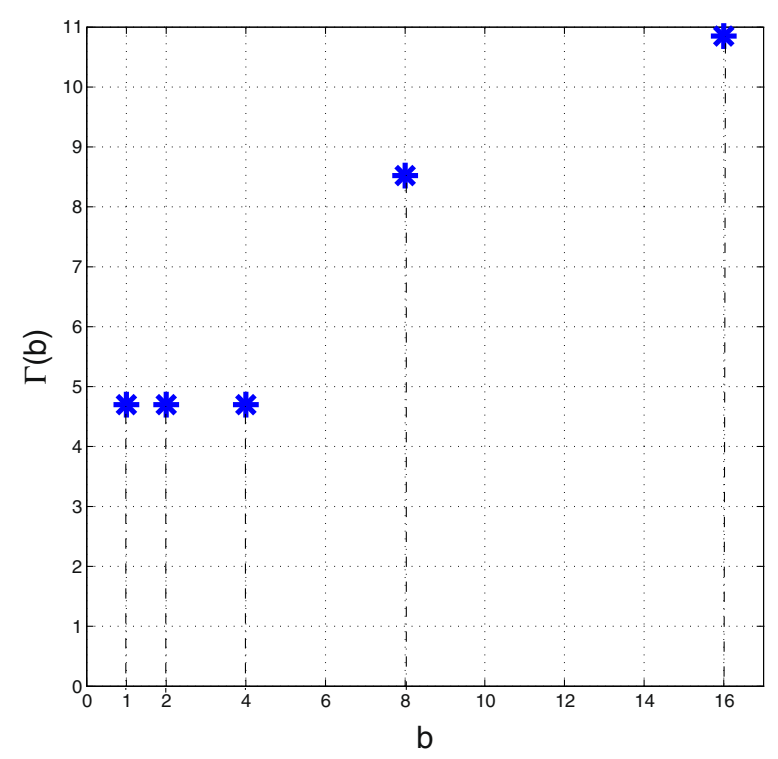

Figure 7 Function $\Gamma(b)$ with $N_{p}=64, N_{u}=4, L=4$, and $\sigma^{2}(1)=0.25, \sigma^{2}(2)=0.5, \sigma^{2}(3)=0.125, \sigma^{2}(4)=0.3$. For any $b^{*} \in \beta^{*}=\{1,2,4\}$, we have $\Gamma\left(b^{*}\right)=(0.25+0.5+0.125+0.3) \frac{64}{4^{2}}=4.7$.

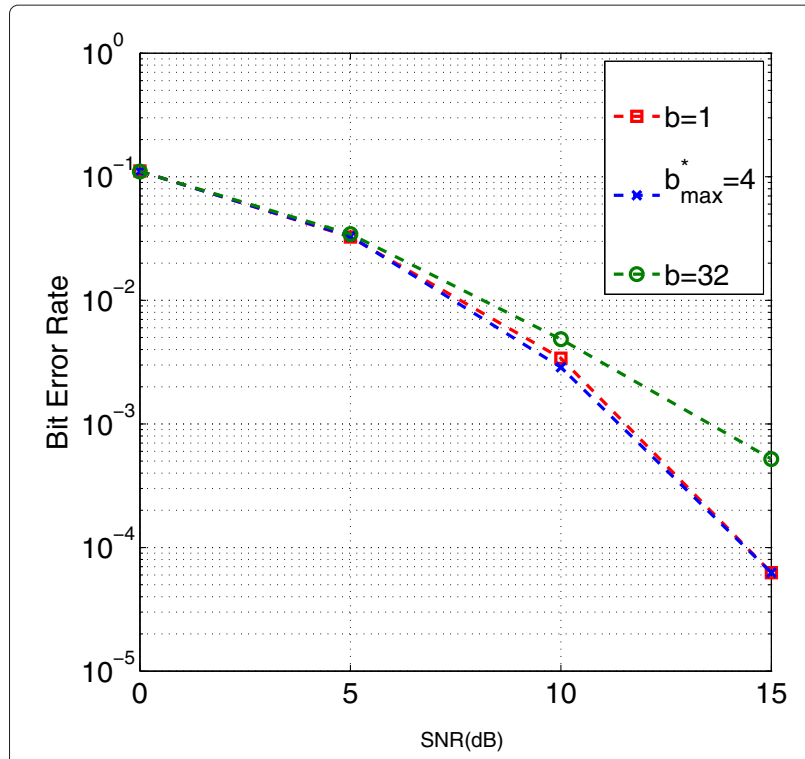

Figure 8 Bit error rate for a SC-FDMA system without CFO for the scenario $N_{p}=64, N_{u}=2$, and $L=8$, and $\sigma_{h}^{(1) 2}=\sigma_{h}^{(2) 2}$. The optimal block-sizes are $b^{*} \in \beta^{*}=\{1,2,4\}$.

The theoretical results are confirmed since the optimal block-sizes are in $\beta^{*}=\{1,2,4,8\}$.

In Figure 11, we evaluate the outage probability $P_{\text {out }}$ in the SC-FDMA system without CFO for the scenario $N_{u}=4, L=4$, and $R=1 \mathrm{bits} / \mathrm{s} / \mathrm{Hz}$. We observe that the optimal block-sizes are the ones that correspond to the outage probabilities which have a higher

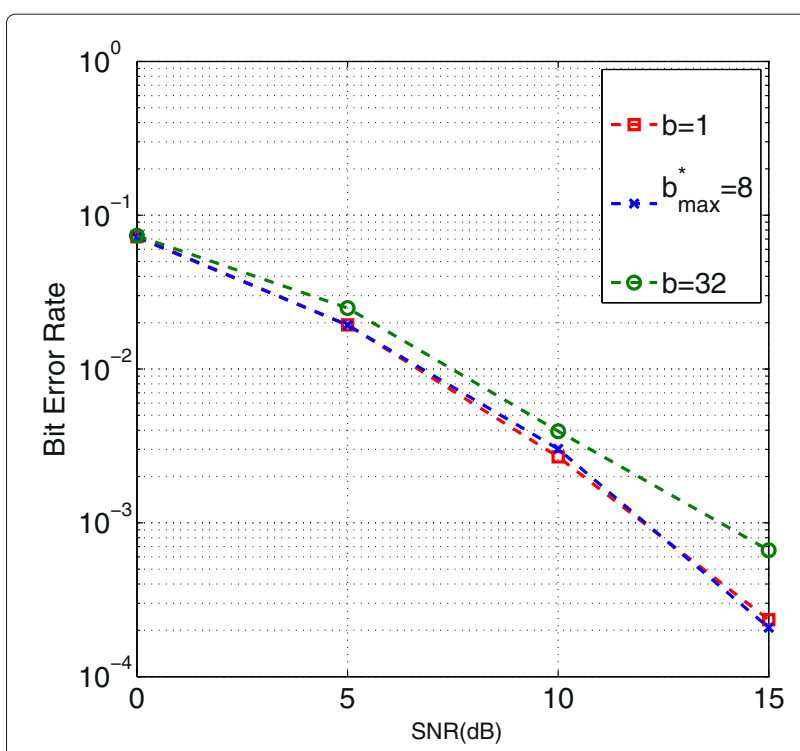

Figure 9 Bit error rate for a SC-FDMA system without CFO for the scenario $N_{p}=64, N_{u}=2, L=4$, and $\sigma_{h}^{(2) 2}=2 \sigma_{h}^{(1) 2}$. The optimal block-sizes are $b^{*} \in \beta^{*}=\{1,2,4,8\}$. 


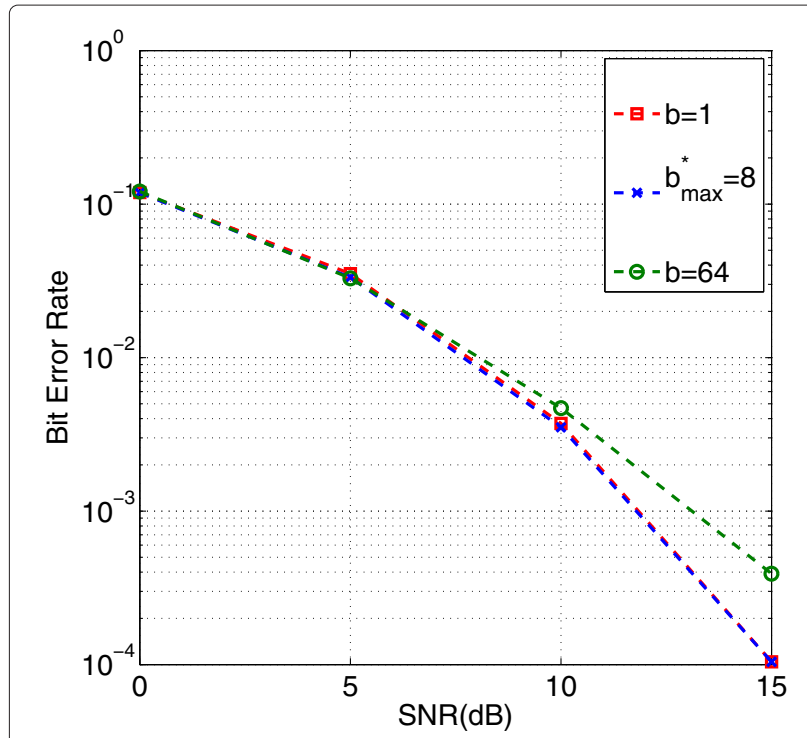

Figure 10 Bit error rate for a SC-FDMA system without CFO for the scenario $N_{p}=128, N_{u}=2, L=8$, and exponential power delay profile. The optimal block-sizes are $b^{*} \in \beta^{*}=\{1,2,4,8\}$.

decreasing rate as a function of the SNR. We see that the curves with $b^{*} \in \beta^{*}=\{1,2,4\}$ (in this case $\left.b_{\max }^{*}=\frac{64}{4 \times 4}=4\right)$ are overlapped and they represent the lower outage probability. These block-sizes are the same that minimize the sum-correlation function (see Figure 7).

In Figure 12, we plot the outage probability for the SCFDMA system without CFO for the scenario $N_{p}=64$,

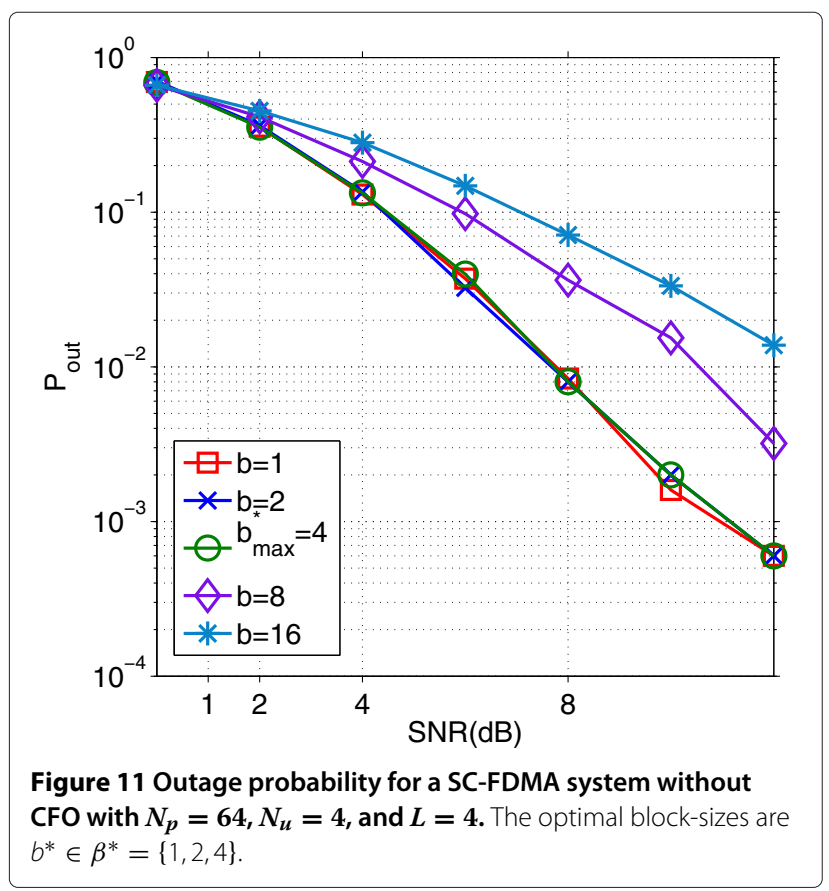

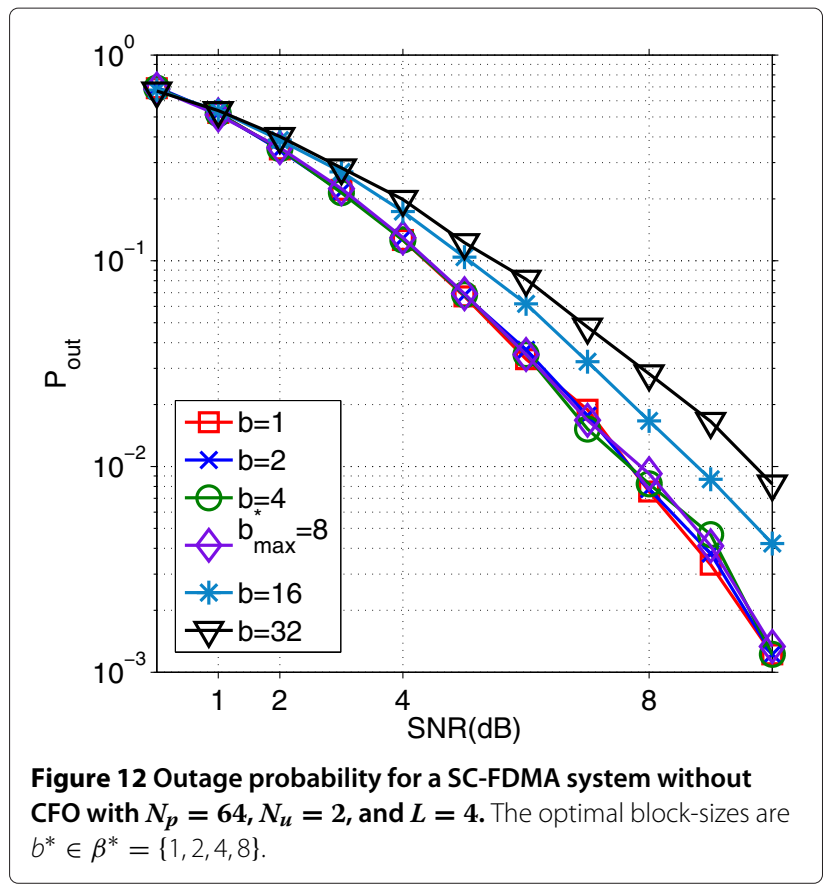

$N_{u}=2, L=4$, and $R=1 \mathrm{bits} / \mathrm{s} / \mathrm{Hz}$ so that $b_{\max }^{*}=\frac{64}{2 \times 4}=$ 8 . In this case in which the subcarriers are correlated, we observe that the curves with $b^{*} \in \beta^{*}=\{1,2,4,8\}$ have a higher diversity.

Many others simulations, changing the values of the parameters (in particular, $N_{p}$ and $L$ ), have been performed, and similar observations were made. Moreover, we have done simulations choosing the following as a performance metric:

$$
\tilde{P}_{\text {out }, b}=1-\prod_{u=1}^{N_{u}}\left(1-P_{\text {out }, b}^{(u)}\right) .
$$

The same observation can be made with this outage metric.

\section{Robustness to CFO}

In this section, we analyze the case of SC-FDMA systems with CFO and the effect of CFO on the optimal block-size. We define the sum-correlation function and we show its robustness to CFO.

We start by describing in details the system model.

\subsection{System model}

If the system undergoes CFOs, the signal at the input of the receiver DFT is given in (25), and it was introduced in [10], 


$$
\begin{aligned}
& \left(\begin{array}{c}
y_{N_{p}-1} \\
\vdots \\
y_{0} \\
\vdots \\
y_{-L}
\end{array}\right)=\sum_{u=1}^{N_{u}}\left(\begin{array}{ccccc}
h_{0}^{(u)} & \cdots & h_{L-1}^{(u)} & & \\
& & & & 0 \\
& \ddots & & \ddots & \\
0 & & & & \\
& & h_{0}^{(u)} & \cdots & h_{L-1}^{(u)}
\end{array}\right) \\
& \times\left(\begin{array}{ccc}
\delta_{\left(N_{p}+L-1\right)}^{(u)} & & \\
& & 0 \\
& \ddots & \\
0 & & \\
& & \delta_{0}^{(u)}
\end{array}\right)\left(\begin{array}{c}
a_{N_{p}-1}^{(u)} \\
\vdots \\
a_{0}^{(u)} \\
\vdots \\
a_{-L}^{(u)}
\end{array}\right)+\left(\begin{array}{c}
n_{N_{p}-1} \\
\vdots \\
n_{0} \\
\vdots \\
n_{-L}
\end{array}\right)
\end{aligned}
$$

The diagonal elements $\delta_{k}^{(u)}$ are the frequency shift coefficients given by $\delta_{k}^{(u)}=e^{\frac{j 2 \pi k \delta f_{c}^{(u)} T}{N_{p}}}, k \in\left\{0, \ldots, N_{p}+\right.$ $L-1\}$, where $\frac{\delta f_{c}^{(u)}}{N_{p}}$ is the normalized CFO of user $u$.

By discarding the cyclic prefix symbols and rearranging the terms in (25), we have

$$
\begin{aligned}
\left(\begin{array}{c}
y_{N_{p}-1} \\
\vdots \\
y_{0}
\end{array}\right)= & \sum_{u=1}^{N_{u}} \mathbf{h}_{\text {circ }}^{(u)}\left(\begin{array}{ccc}
\delta_{\left(N_{p}+L-1\right)}^{(u)} & & 0 \\
0 & \ddots & \\
& & \delta_{L}^{(u)}
\end{array}\right) \\
& \times\left(\begin{array}{c}
a_{N_{p}-1}^{(u)} \\
\vdots \\
a_{0}^{(u)}
\end{array}\right)+\left(\begin{array}{c}
n_{N_{p}-1}^{(u)} \\
\vdots \\
n_{0}
\end{array}\right) .
\end{aligned}
$$

The received signal at the BS after the DFT is

$$
\mathbf{r}_{\mathrm{CFO}}=\sum_{u=1}^{N_{u}} \mathbf{H}^{(u)} \boldsymbol{\Delta}^{(u)} \boldsymbol{\Pi}_{b}^{(u)} \mathbf{x}^{(u)}+\hat{\mathbf{n}}
$$

where the $N_{p} \times N_{p}$ matrix $\boldsymbol{\Delta}^{(u)}=\mathbf{F} \delta^{(u)} \mathbf{F}^{-1}$ represents the effect of CFO on the interference among subcarriers. In particular, we have the $(l, k)$ element of $\boldsymbol{\Delta}^{(u)}$ :

$$
\begin{aligned}
\Delta_{\ell, k}^{(u)} & =\frac{1}{N_{p}} \sum_{i=0}^{N_{p}-1} e^{j 2 \pi i \delta f / N_{p}} e^{-j 2 \pi i(\ell-k) / N_{p}} \\
& =\frac{1}{N_{p}} \frac{\sin (\pi(\delta f+k-\ell))}{\sin \left(\frac{\pi}{N_{p}}(\delta f+k-\ell)\right)} e^{\pi j\left(1-\frac{1}{N_{p}}\right)(\delta f+k-\ell)}
\end{aligned}
$$

In the sequel, we denote $\tilde{\mathbf{H}}^{(u)} \triangleq \mathbf{H}^{(u)} \boldsymbol{\Delta}^{(u)}$, which is no longer a diagonal matrix.

\subsection{Diversity versus CFO in subcarrier allocation}

We consider the following inter-carrier correlation function:

$$
\begin{aligned}
& \Gamma_{u, m}^{\mathrm{CFO}}(b, \delta f) \triangleq \sum_{c_{u} \in \mathcal{C}_{u}} \mathbb{E}\left[H_{m}^{(u)} \Delta_{m, m}[1, \ldots, 1] \tilde{\mathbf{H}}_{c_{u}}^{(u) \dagger}\right] \\
& =\sum_{c_{u} \in \mathcal{C}_{u}} \sum_{k \in \mathcal{C}_{u}} \mathbb{E}\left[H_{m}^{(u)} H_{\mathcal{c}_{u}}^{(u) *}\right] \Delta_{m, m} \Delta_{c_{u}, k}^{*} \\
& =\sum_{k \in \mathcal{C}_{u}} \mathbb{E}\left[\left|H_{m}^{(u)}\right|^{2}\right] \Delta_{m, m} \Delta_{m, k}^{*} \\
& +\sum_{\substack{c_{u} \in \mathcal{C}_{u} \\
c_{u} \neq m}} \sum_{k \in \mathcal{C}_{u}} \mathbb{E}\left[H_{m}^{(u)} H_{\mathcal{C}_{u}}^{(u) *}\right] \Delta_{m, m} \Delta_{c_{u}, k}^{*} \\
& =\mathbb{E}\left[\left|H_{m}^{(u)}\right|^{2}\right]\left|\Delta_{m, m}\right|^{2}+\sum_{\substack{k \in \mathcal{C}_{u} \\
k \neq m}} \mathbb{E}\left[\left|H_{m}^{(u)}\right|^{2}\right] \Delta_{m, m} \Delta_{m, k}^{*}+ \\
& +\sum_{\substack{c_{u} \in \mathcal{C}_{u} \\
c_{u} \neq m}} \mathbb{E}\left[H_{m}^{(u)} H_{c_{u}}^{(u) *}\right] \Delta_{m, m} \Delta_{c_{u}, c_{u}}^{*} \\
& +\sum_{\substack{c_{u} \in \mathcal{C}_{u} \\
c_{u} \neq m}} \sum_{\substack{k \in \mathcal{C}_{u} \\
k \neq c_{u}}} \mathbb{E}\left[H_{m}^{(u)} H_{c_{u}}^{(u) *}\right] \Delta_{m, m} \Delta_{c_{u}, k}^{*} \\
& =\sigma_{h}^{(u) 2} \frac{L_{h}}{N_{p}}\left[\frac{1}{N_{p}^{2}} \frac{\sin ^{2}(\pi \delta f)}{\sin ^{2}\left(\frac{\pi}{N_{p}} \delta f\right)}\right. \\
& +\sum_{\substack{k \in \mathcal{C}_{u} \\
k \neq m}} \frac{1}{N_{p}^{2}} e^{-\pi j\left(1-\frac{1}{N_{p}}\right)(k-m)} \frac{\sin (\pi \delta f)}{\sin \left(\frac{\pi}{N_{p}} \delta f\right)} \\
& \left.\times \frac{\sin (\pi(\delta f+k-m))}{\sin \left(\frac{\pi}{N_{p}}(\delta f+k-m)\right)}\right]+ \\
& +\frac{\sigma_{h}^{(u) 2}}{N_{p}} \sum_{\substack{c_{u} \in \mathcal{C}_{u} \\
c_{u} \neq m}} e^{-\pi j \frac{\left(L_{h}-1\right)}{N_{p}}\left(m-c_{u}\right)} \frac{\sin \left[\pi \frac{L_{h}}{N_{p}}\left(m-c_{u}\right)\right]}{\sin \left[\frac{\pi}{N_{p}}\left(m-c_{u}\right)\right]} \times \\
& \times\left[\frac{1}{N_{p}^{2}} \frac{\sin ^{2}(\pi \delta f)}{\sin ^{2}\left(\frac{\pi}{N_{p}} \delta f\right)}+\sum_{\substack{k \in \mathcal{C}_{u} \\
k \neq c_{u}}} \frac{1}{N_{p}^{2}} e^{-\pi j\left(1-\frac{1}{N_{p}}\right)\left(k-c_{u}\right)}\right. \\
& \left.\times \frac{\sin (\pi \delta f)}{\sin \left(\frac{\pi}{N_{p}} \delta f\right)} \frac{\sin \left(\pi\left(\delta f+k-c_{u}\right)\right)}{\sin \left(\frac{\pi}{N_{p}}\left(\delta f+k-c_{u}\right)\right)}\right]
\end{aligned}
$$

where $m \in \mathcal{C}_{u}$ is the reference subcarrier, $\delta f$ represents the CFO of user $u$, and $\tilde{\mathbf{H}}_{c_{u}}^{(u)}=\left(H_{c_{u}}^{(u)} \Delta_{c_{u}, 1}, \ldots, H_{c_{u}}^{(u)} \Delta_{c_{u}, N_{p}}\right)$ represents the $c_{u}$-th row of the matrix $\tilde{\mathbf{H}}^{(u)}$.

We define the sum-correlation metric as follows:

$$
\Gamma^{C F O}(b, \delta f)=\sum_{u=1}^{N_{u}} \sum_{m \in \mathcal{C}_{u}} \Gamma_{u, m}^{\mathrm{CFO}}(b, \delta f) .
$$

In the following, we provide an approximation of the correlation function $\Gamma_{m, u}^{\mathrm{CFO}}(b, \delta f)$ in which the dependance on the CFO values $\delta f$ is taken into account. In particular, 
we consider the second order Taylor approximation of $\Gamma_{m, u}^{\mathrm{CFO}}(b, \delta f)$ when $\delta f \rightarrow 0$

$$
\begin{aligned}
\Gamma_{m, u}^{\mathrm{CFO}}(b, \delta f) \approx & \Gamma_{m, u}^{\mathrm{CFO}}(b, 0)+\frac{d \Gamma_{m, u}^{\mathrm{CFO}}}{d \delta f}(b, 0) \delta f \\
& +\frac{1}{2} \frac{d^{2} \Gamma_{m, u}^{\mathrm{CFO}}}{d(\delta f)^{2}}(b, 0)(\delta f)^{2} .
\end{aligned}
$$

This first term $\Gamma_{m, u}^{\mathrm{CFO}}(b, 0)$ is given by

$$
\begin{aligned}
\Gamma_{u, m}^{C F O}(b, 0)= & \sigma_{h}^{(u) 2} \frac{L_{h}}{N_{p}}\left[\frac{1}{N_{p}^{2}} N_{p}^{2}+\frac{1}{N_{p}^{2}} \sum_{\substack{k \in \mathcal{C}_{u} \\
k \neq m}} e^{-\pi j\left(1-\frac{1}{N_{p}}\right)(k-m)} N_{p} \times 0\right]+ \\
& +\frac{\sigma_{h}^{(u) 2}}{N_{p}} \sum_{\substack{c_{u} \in \mathcal{C}_{u} \\
c_{u} \neq m}}^{-\pi j \frac{\left(L_{h}-1\right)}{N_{p}}\left(m-c_{u}\right)} \frac{\sin \left[\pi \frac{L_{h}}{N_{p}}\left(m-c_{u}\right)\right]}{\sin \left[\frac{\pi}{N_{p}}\left(m-c_{u}\right)\right]} \times \\
& \times\left[\frac{1}{N_{p}^{2}} N_{p}^{2}+\frac{1}{N_{p}^{2}} \sum_{\substack{k \in \mathcal{C}_{u} \\
k \neq c_{u}}} e^{-\pi j\left(1-\frac{1}{N_{p}}\right)\left(k-c_{u}\right)} N_{p} \times 0\right] \\
= & \sigma_{h}^{(u) 2} \frac{L_{h}}{N_{p}}+\frac{\sigma_{h}^{(u) 2}}{N_{p}} \sum_{\substack{c_{u} \in \mathcal{C}_{u} \\
c_{u} \neq m}} e^{-\pi j \frac{\left(L_{h}-1\right)}{N_{p}}\left(m-c_{u}\right)} \\
& \frac{\sin \left[\pi \frac{L_{h}}{N_{p}}\left(m-c_{u}\right)\right]}{\sin \left[\frac{\pi}{N_{p}}\left(m-c_{u}\right)\right]} .
\end{aligned}
$$

Indeed, this expression corresponds exactly to $\Gamma_{m, u}(b)$, i.e., the sum-correlation function in the case without $\mathrm{CFO}$ in (6).

The first derivative of $\Gamma_{m, u}^{\mathrm{CFO}}(b, \delta f)$ with respect to $\delta f$ computed in $(b, 0)$ is

$$
\begin{aligned}
& \frac{d \Gamma_{m, u}^{\mathrm{CFO}}}{d \delta f}(b, 0)=\sigma_{h}^{(u) 2} \frac{L_{h}}{N_{p}}\left[\sum_{\substack{k \in \mathcal{C}_{u} \\
k \neq m}} \frac{1}{N_{p}^{2}} e^{-\pi j\left(1-\frac{1}{N_{p}}\right)(k-m)}\right. \\
& \left.\times \frac{\pi N_{p} \cos (\pi(k-m))}{\sin \left(\frac{\pi}{N_{p}}(k-m)\right)}\right]+ \\
& +\frac{\sigma_{h}^{(u) 2}}{N_{p}} \sum_{\substack{c_{u} \in \mathcal{C}_{u} \\
c_{u} \neq m}} e^{-\pi j \frac{\left(L_{h}-1\right)}{N_{p}}\left(m-c_{u}\right)} \frac{\sin \left[\pi \frac{L_{h}}{N_{p}}\left(m-c_{u}\right)\right]}{\sin \left[\frac{\pi}{N_{p}}\left(m-c_{u}\right)\right]} \times \\
& \times\left[\sum_{\substack{k \in \mathcal{C}_{u} \\
k \neq \mathcal{C}_{u}}} \frac{1}{N_{p}^{2}} e^{-\pi j\left(1-\frac{1}{N_{p}}\right)\left(k-c_{u}\right)} \frac{\pi N_{p} \cos \left(\pi\left(k-c_{u}\right)\right)}{\sin \left(\frac{\pi}{N_{p}}\left(k-c_{u}\right)\right)}\right]
\end{aligned}
$$

The second derivative of $\Gamma_{m, u}^{\mathrm{CFO}}(b, \delta f)$ computed in $(b, 0)$ is

$$
\begin{aligned}
\frac{1}{2} \frac{d^{2} \Gamma_{m, u}^{\mathrm{CFO}}}{d(\delta f)^{2}}(b, 0)= & \frac{1}{2} \sigma_{h}^{(u) 2} \frac{L_{h}}{N_{p}}\left[\frac{2 \pi^{2}\left(1-N_{p}^{2}\right)}{N_{p}^{2}}-\frac{1}{N_{p}^{2}} \sum_{\substack{k \in \mathcal{C}_{u} \\
k \neq m}} e^{-\pi j\left(1-\frac{1}{N_{p}}\right)(k-m)}\right. \\
& \left.\times \frac{2 \pi^{2} \cos \left(\frac{\pi}{N_{p}}(k-m)\right)}{\sin ^{2}\left(\frac{\pi}{N_{p}}(k-m)\right)}\right]+ \\
& +\frac{1}{2} \frac{\sigma_{h}^{(u) 2}}{N_{p}} \sum_{\substack{c_{u} \in \mathcal{C}_{u} \\
c_{u} \neq m}} e^{-\pi j \frac{\left(L_{h}-1\right)}{N_{p}}\left(m-c_{u}\right)} \\
& \times \frac{\sin \left[\pi \frac{L_{h}}{N_{p}}\left(m-c_{u}\right)\right]}{\sin \left[\frac{\pi}{N_{p}}\left(m-c_{u}\right)\right]} \times \\
& \times\left[\frac{2 \pi^{2}\left(1-N_{p}^{2}\right)}{N_{p}^{2}}-\frac{1}{N_{p}^{2}} \sum_{\substack{k \in \mathcal{C}_{u} \\
k \neq c_{u}}} e^{-\pi j\left(1-\frac{1}{N_{p}}\right)\left(k-c_{u}\right)}\right. \\
& \left.\times \frac{2 \pi^{2} \cos \left(\frac{\pi}{N_{p}}\left(k-c_{u}\right)\right)}{\sin ^{2}\left(\frac{\pi}{N_{p}}\left(k-c_{u}\right)\right)}\right]
\end{aligned}
$$

Therefore, we have

$$
\begin{aligned}
& \Gamma_{m, u}^{\mathrm{CFO}}(b, \delta f) \approx \frac{\sigma_{h}^{(u) 2}}{N_{p}} L_{h}+\frac{\sigma_{h}^{(u) 2}}{N_{p}} \sum_{c_{u} \in \mathcal{C}_{u}} \frac{\sin \left[\frac{\pi L_{h}}{N_{p}}\left(m-c_{u}\right)\right]}{\left.\sin \left(m-c_{u}\right)\right]} \times e^{-\pi j \frac{\left(L_{h}-1\right)}{N_{p}}\left(m-c_{u}\right)}+ \\
& +\left\{\sigma_{h}^{(u) 2} \frac{L_{h}}{N_{p}}\left[\sum_{\substack{k \in \mathcal{C}_{u} \\
k \neq m}} \frac{1}{N_{p}} e^{-\pi i\left(1-\frac{1}{N_{p}}\right)(k-m)} \frac{\cos (\pi(k-m))}{\sin \left(\frac{\pi}{N_{p}}(k-m)\right)}\right]+\right. \\
& +\frac{\sigma_{h}^{(u) 2}}{N_{p}} \sum_{\substack{c_{u} \in \mathcal{C}_{u} \\
c_{u} \neq m}} e^{-\pi j \frac{\left(\mathcal{L}_{h}-1\right)}{N_{p}}\left(m-c_{u}\right)} \frac{\sin \left[\pi \frac{L_{h}}{N_{p}}\left(m-c_{u}\right)\right]}{\sin \left[\frac{\pi}{N_{p}}\left(m-c_{u}\right)\right]} \times \\
& \left.\times\left[\sum_{\substack{k \in \mathcal{C}_{u} \\
k \neq c_{u}}} \frac{1}{N_{p}} e^{-\pi j\left(1-\frac{1}{N_{p}}\right)\left(k-c_{u}\right)} \frac{\cos \left(\pi\left(k-c_{u}\right)\right)}{\sin \left(\frac{\pi}{N_{p}}\left(k-c_{u}\right)\right)}\right]\right\} \pi \delta \\
& +\left\{\sigma _ { h } ^ { ( u ) 2 } \frac { L _ { h } } { N _ { p } } \left[\frac{\left(1-N_{p}^{2}\right)}{N_{p}^{2}}-\frac{1}{N_{p}^{2}} \sum_{\substack{k \in \mathcal{C}_{u} \\
k \neq m}} e^{-\pi j\left(1-\frac{1}{N_{p}}\right)(k-m)}\right.\right. \\
& \left.\times \frac{\cos \left(\frac{\pi}{N_{p}}(k-m)\right)}{\sin ^{2}\left(\frac{\pi}{N_{p}}(k-m)\right)}\right]+ \\
& +\frac{\sigma_{h}^{(u) 2}}{N_{p}} \sum_{\substack{c_{u} \in \mathcal{C}_{u} \\
c_{u} \neq m}} e^{-\pi j \frac{\left(L_{h}-1\right)}{N_{p}}\left(m-c_{u}\right)} \frac{\sin \left[\pi \frac{L_{h}}{N_{p}}\left(m-c_{u}\right)\right]}{\sin \left[\frac{\pi}{N_{p}}\left(m-c_{u}\right)\right]} \times \\
& \times\left[\frac{\left(1-N_{p}^{2}\right)}{N_{p}^{2}}-\frac{1}{N_{p}^{2}} \sum_{\substack{k \in \mathcal{C}_{u} \\
k \neq c_{u}}} e^{-\pi j\left(1-\frac{1}{N_{p}}\right)\left(k-c_{u}\right)}\right. \\
& \left.\left.\times \frac{\cos \left(\frac{\pi}{N_{p}}\left(k-c_{u}\right)\right)}{\sin ^{2}\left(\frac{\pi}{N_{p}}\left(k-c_{u}\right)\right)}\right]\right\} \pi^{2}(\delta f)^{2} .
\end{aligned}
$$


We observe in the above approximation the presence of the predominant term represented by the sum-correlation $\Gamma_{m, u}(b)$ without CFO and the first and the second derivatives of $\Gamma_{m, u}^{\mathrm{CFO}}(b, \delta f)$ which are multiplied by the CFO value $\delta f$ and $\delta f^{2}$, respectively. This last terms may result in a different solution for the optimal block-size or block-sizes that optimize the sum-correlation function than the solution for the case with no CFO. We observe that the first and the second derivatives represent a complex function that implicitly depends on $b$. Finding the optimal blocksize or block-sizes in an analytical manner, as done in the case with no CFO, seems very difficult if at all possible and is left for future investigation.

When the system undergoes CFO, the carrier correlation and CFO affect the system performance simultaneously. We have proposed in [12] the largest optimal block-size $b_{\max }^{*}$ as the unique optimal block-size: Since CFO yields a diversity loss, in the presence of moderate values of CFO, the optimal block-size allocation is $b_{\text {max }}^{*}$. We have found that the optimal block-sizes that achieve maximum diversity are the ones that minimize the correlation between subcarriers. Moreover, larger blocksizes are preferable to combat the effect of ICI. Also, since $b_{\max }^{*}$ is the largest block-size between the ones minimizing the correlation, it is also the one that minimizes the negative effects caused by the presence of CFO. Therefore, $b_{\max }^{*}$ represents a good tradeoff between diversity and CFO. Moreover, the observation is validated also by numerical simulations illustrated in the next subsection.

\subsection{Numerical results: CFO impact}

In Figure 13, we plot the correlation $\Gamma^{\mathrm{CFO}}(b, \delta f)$ for the scenario: $N_{p}=64, N_{u}=2, L_{h}=8$, and $\sigma_{h}^{(1) 2}=$ $0.25, \sigma_{h}^{(2) 2}=0.5$. The considered CFO values are $\delta f \in$ $\{0.1,0.2,0.3,0.4\}$. We observe that $b_{\max }^{*}=4$ is the blocksize that achieves the minimum value of the correlation function $\Gamma^{\mathrm{CFO}}(b, \delta f)$, validating our conjectured optimal block-size.

In the next two simulations, we use BPSK modulation and $N p=128, N u=2$, and $L=8$. Figure 14 illustrates the bit error rate (BER) curves for a SC-FDMA system with CFO independently and uniformly generated for each user in $[0,0.03]$. We observe that for these low CFOs we have the optimal block-sizes given by $\beta^{*}=\{1,2,4,8\}$. Figure 15 illustrates the BER curves for a SC-FDMA system with CFO independently and uniformly generated for each user in $[0,0.1]$. We observe that, in this case, we have a unique optimal block-size given by $\beta_{\max }^{*}=8$. This validates our observations, i.e., when the CFO's values are increasing, the best tradeoff between diversity and CFO is represented by the largest block-size of our proposed set $b_{\max }^{*}$.

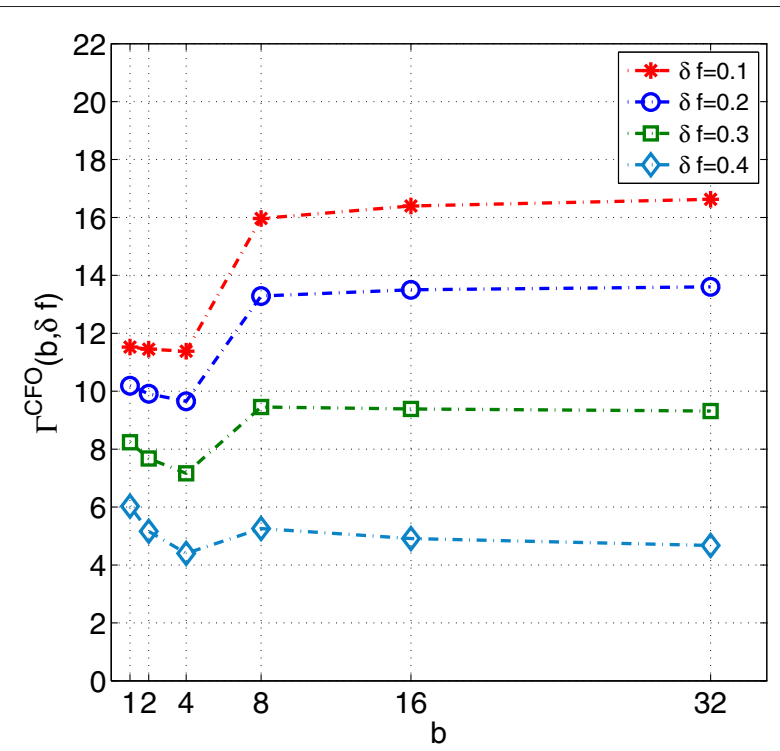

Figure 13 The correlation function $\Gamma^{C F O}(b, \delta f)$ as function of $b$ for the scenario: $N_{p}=64, N_{u}=2, L_{h}=8$, and $\sigma_{h}^{(1) 2}=0.25$, $\boldsymbol{\sigma}_{\boldsymbol{h}}^{(2) 2}=\mathbf{0 . 5}, \boldsymbol{\delta} \boldsymbol{f} \in\{\mathbf{0 . 1}, \mathbf{0 . 2}, \mathbf{0 . 3}, \mathbf{0 . 4}\}$. The optimal block-size minimizing the correlation function is $b_{\max }^{*}=\frac{N_{p}}{L N_{u}}=4$.

In Figure 16, we use BPSK modulation in the following scenario: $N_{p}=64, N_{u}=2$, and $L=4$. We consider the same model proposed in [11] where one symbol is spread over all subcarriers. The CFO is independently and uniformly generated for each user in $[0,0.1]$. We observe that

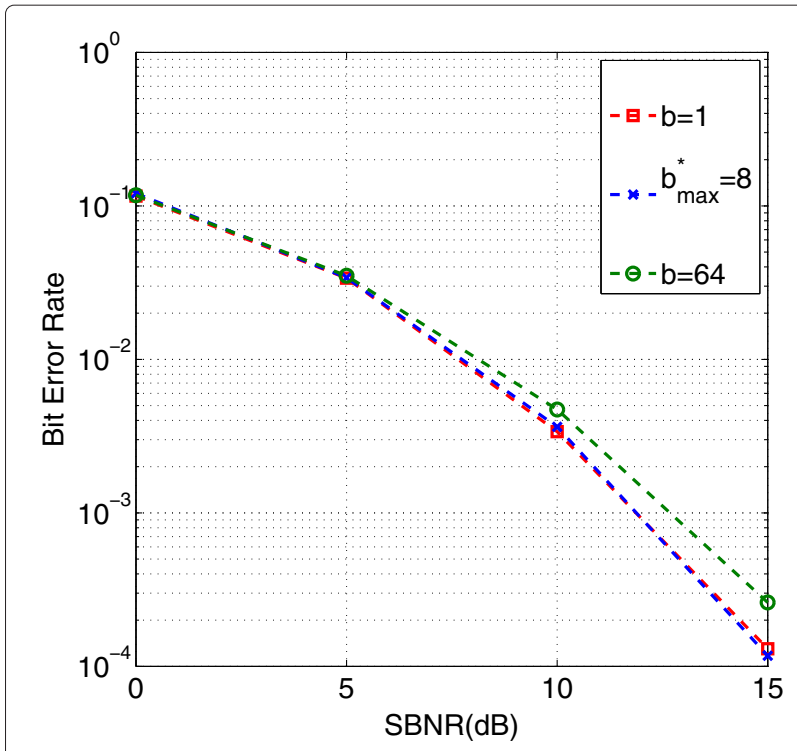

Figure 14 Bit error rate for a SC-FDMA system with CFO for the scenario $N_{p}=128, N_{u}=\mathbf{2}$, and $L=8$. The CFO of each user is independently uniformly generated in $[0,0.03]$. The optimal block-sizes are $b^{*} \in \beta^{*}=\{1,2,4,8\}$. 


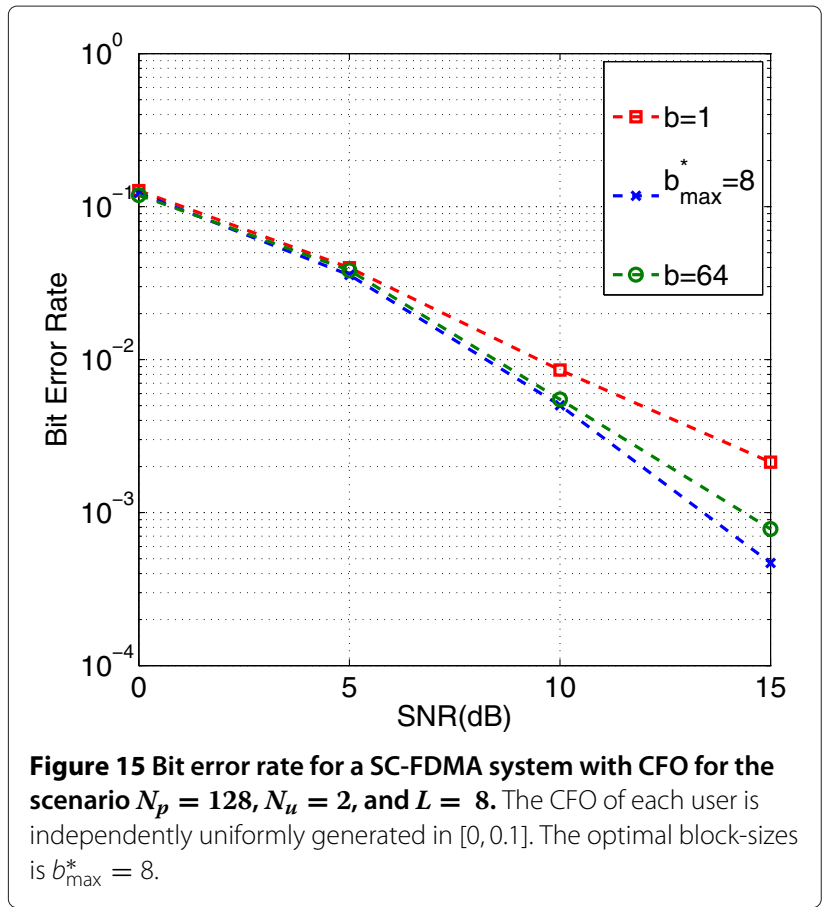

the optimal block-sizes are in $\beta^{*}=\{1,2,4,8\}$ for the BER which confirms that our analysis is valid for the model proposed in [11].

In Figure 17, we use BPSK, $N_{p}=64, N_{u}=2$, and $L=8$ and an exponential power delay profile. The CFO is independently and uniformly generated for each user

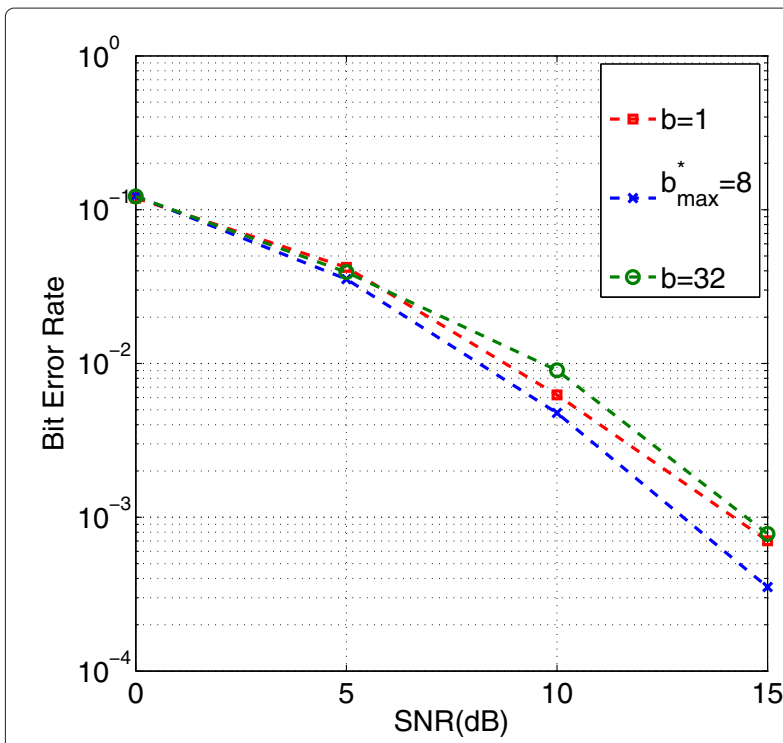

Figure 16 Bit error rate for a SC-FDMA system with CFO for the scenario $N_{p}=64, N_{u}=\mathbf{2}$, and $L=4$. The CFO is independently and uniformly generated for each user in $[0,0.1]$. The optimal block-sizes are $b^{*} \in \beta^{*}=\{1,2,4,8\}$.

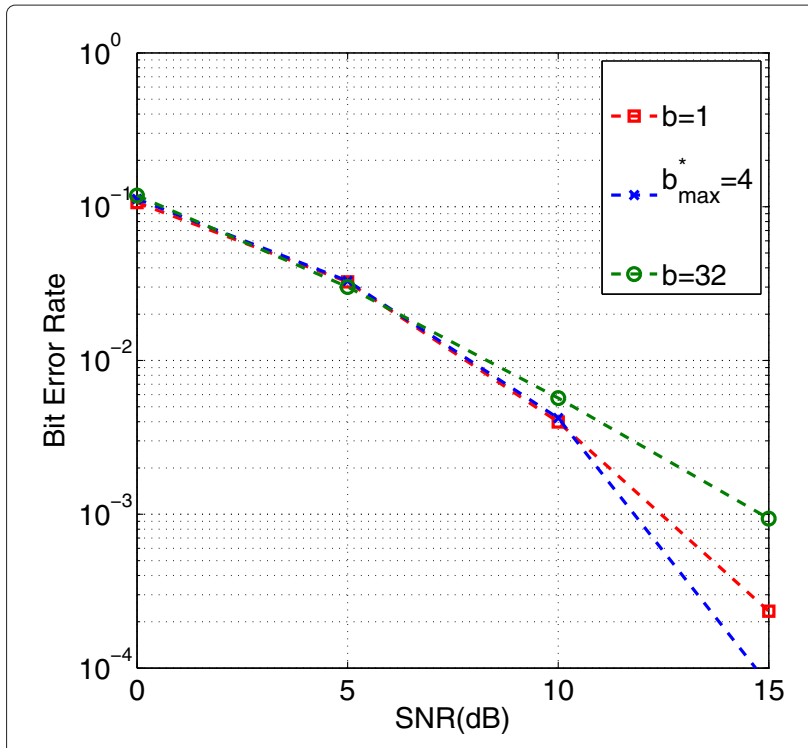

Figure 17 Bit error rate for a SC-FDMA system with CFO for the scenario $\boldsymbol{N}_{\boldsymbol{p}}=\mathbf{6 4}, \boldsymbol{N}_{\boldsymbol{u}}=\mathbf{2}$, and $\boldsymbol{L}=\mathbf{8}$. The CFO is independently and uniformly generated for each user in $[0,0.05]$. The optimal block-sizes are $b^{*} \in \beta^{*}=\{1,2,4\}$.

in $[0,0.05]$. The set of optimal block-sizes given by $\beta^{*}=$ $\{1,2,4\}$ as shown in the figure.

For different and larger CFO values, as considered in [18], we notice that an error floor is obtained due to the effect of CFO interference. Thus, in such cases, optimizing the block-size is not very relevant as all possibilities obtain such poor results in terms of BER.

In the next simulation, we consider the following scenario: $N_{p}=64, N_{u}=4$, and $L=8$. In the Figure 18, we plot the outage probability of an SC-FDMA system with CFO (marker lines) against the outage probability of the SC-FDMA system without CFO (dashed lines). The CFO for each user is independently uniformly generated in $\delta f \in$ $[0,0.01]$, and the rate $R$ is taken equal to $1 \mathrm{bits} / \mathrm{s} / \mathrm{Hz}$. We can see that the curves in the CFO case fit very well the outage probability curves without CFO. In particular, they appear in a decreasing order of block-size. This validates our analytical analysis on the approximation of the CFO sum-correlation function to the case without $\mathrm{CFO}$ when the CFO goes to zero. Moreover, we observe that in the two cases we have the same optimal block-sizes set, given by $\beta^{*}=\{1,2\}$.

\section{Conclusions}

In this work, we have provided the analytical expression of the set of optimal sizes of subcarrier blocks for SCFDMA uplink systems without CFO and without channel state information. These optimal block-sizes allow us to minimize the sum-correlation between subcarriers and to achieve maximum diversity gain. We have also provided 


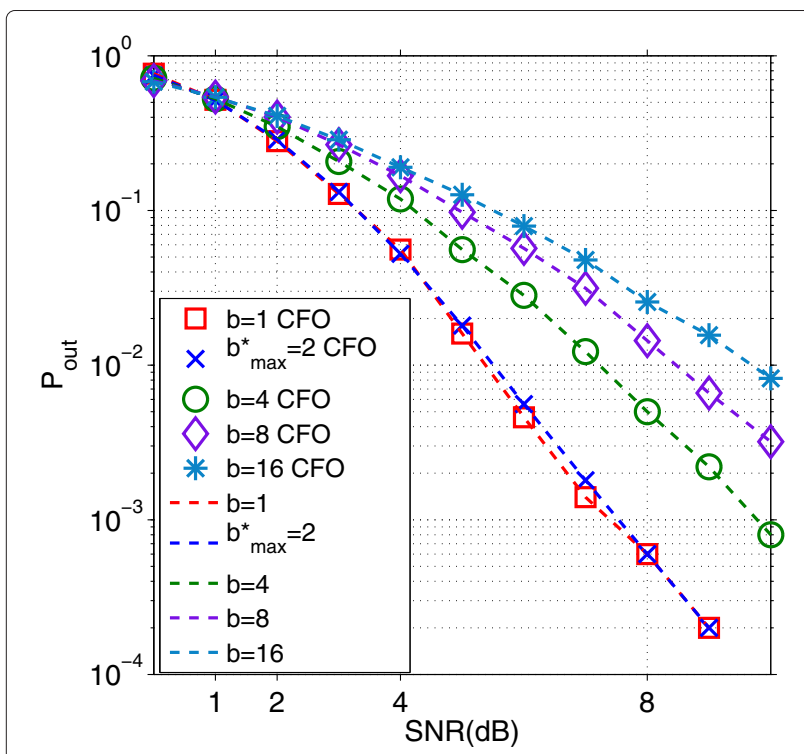

Figure 18 Outage probability for a SC-FDMA system with and without CFO. Outage probability for a SC-FDMA system without CFO (appearing in decreasing order of block-size from up to down) and with CFO (marker lines) for the scenario $\boldsymbol{N}_{\boldsymbol{p}}=\mathbf{6 4}, \boldsymbol{N}_{\boldsymbol{u}}=\mathbf{4}$, and $\boldsymbol{L}=\mathbf{8}$. The CFO of each user is independently uniformly generated in $[0,0.01]$. The optimal block-sizes are $b^{*} \in \beta^{*}=\{1,2\}$.

the analytical expression of the sum-correlation between subcarriers induced by SC-FDMA/OFDMA. Moreover, we have found an explicit expression of the largest optimal block-size which minimizes the sum-correlation function depending on the system's parameters: number of subcarriers, number of users, and the cyclic prefix length. Interesting properties of this novel sum-correlation function are also presented.

It turns out that the minimal sum-correlation value depends only on the number of subcarriers, number of users, and the variance of the channel impulse response. We validate via numerical simulations that the set of optimal block-sizes achieving maximum diversity minimizes the outage probability in the case without CFO.

Also, in the case where the system undergoes CFO, we consider a sum-correlation function which is robust to CFO. Robustness is induced by the fact that when the CFO goes to zero, the CFO sum-correlation can be well approximated by the sum-correlation function defined in the case without CFO. Therefore, we propose $b_{\max }^{*}=\frac{N_{p}}{L_{h} N_{u}}$ a good tradeoff between diversity and CFO since it represents the unique optimal block-size that achieves maximum diversity. All these results and observations have been validated via extensive Monte Carlo simulations.

\section{Endnotes}

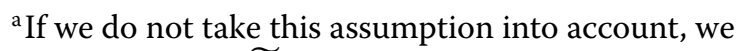
would have to use $\widetilde{N}_{p}=M N_{u}$ instead of $N_{p}$ to denote the actual allocated number of carriers and $\left\lfloor\frac{N_{p}}{N_{u}}\right\rfloor$ instead of $\frac{N_{p}}{N_{u}}$ as the number of carriers per user.

${ }^{b}$ Here we use the word "distance" as synonym of difference and not for Euclidean distance.

\section{Appendix}

Proof of Theorem 1

Proof: From the definition of the set $\mathcal{D}_{b}$, we can write the function $\Gamma(b)$ as follows:

$$
\begin{aligned}
\Gamma(b) & =\sum_{u=1}^{N_{u}} \frac{N_{p}}{N_{u}} \sigma_{h}^{(u) 2} N_{p} \sum_{d \in \mathcal{D}_{b}} f(d) \\
& =\sum_{u=1}^{N_{u}} \frac{\sigma_{h}^{(u) 2}}{N_{u}} \sum_{i=0}^{b-1} \sum_{k=0}^{\frac{N_{p}}{b N_{u}}-1} f\left(k b N_{u}+i\right) \\
& =\sum_{u=1}^{N_{u}} \frac{\sigma_{h}^{(u) 2}}{N_{u}}\left(\sum_{k=0}^{\frac{N_{p}}{b N_{u}}-1} f\left(k b N_{u}\right)+\sum_{i=1}^{b-1} \sum_{k=0}^{\frac{N_{p}}{b N_{u}}-1} f\left(k b N_{u}+i\right)\right) \\
& =\sum_{u=1}^{N_{u}} \frac{\sigma_{h}^{(u) 2}}{N_{u}}\left(L_{h}+\sum_{k=1}^{\frac{N_{p}}{b N_{u}}-1} f\left(k b N_{u}\right)+\sum_{i=1}^{b-1} \sum_{k=0}^{\frac{N_{p}}{b N_{u}}-1} f\left(k b N_{u}+i\right)\right) .
\end{aligned}
$$

First of all, we analyze the term: $\sum_{i=1}^{b-1} \sum_{k=0}^{\frac{N_{p}}{b N_{u}}-1} f\left(k b N_{u}+i\right)$ and, in particular, its $i$ th term

$$
\begin{aligned}
\sum_{k=0}^{\frac{N_{p}}{b N_{u}}-1} f\left(k b N_{u}+i\right) & = \\
& =\sum_{k=0}^{\frac{N_{p}}{b N_{u}}-1} \frac{\sin \left(\pi \frac{L_{h}}{N_{p}}\left(k b N_{u}+i\right)\right)}{\sin \left(\frac{\pi}{N_{p}}\left(k b N_{u}+i\right)\right)} e^{-j \pi \frac{\left(L_{h}-1\right)}{N_{p}}\left(k b N_{u}+i\right)} \\
& =\sum_{k=0}^{\frac{N_{p}}{b N_{u}}-1} \frac{1-e^{-j 2 \pi \frac{L_{h}}{N_{p}} k b N_{u}} e^{-j 2 \pi \frac{L_{h}}{N_{p}} i}}{1-e^{-j 2 \pi \frac{1}{N_{p}} k b N_{u}} e^{-j 2 \pi \frac{1}{N_{p}} i}} \\
& =\sum_{k=0}^{\frac{N_{p}}{b N_{u}}-1} \frac{1-\left(\alpha_{i} z^{k}\right)^{L_{h}}}{1-\alpha_{i} z^{k}}
\end{aligned}
$$

with $\alpha_{i}:=e^{-j 2 \pi \frac{1}{N_{p}} i}$ and $z:=e^{-j 2 \pi \frac{1}{N_{p}} b N_{u}}$. Using the decomposition of a geometric series of radius $\alpha_{i} z^{k}$, we further obtain

$$
\sum_{k=0}^{\frac{N_{p}}{b N_{u}}-1} f\left(k b N_{u}+i\right)=\sum_{k=0}^{\frac{N_{p}}{b N_{u}}-1}\left[\sum_{\ell=0}^{L_{h}-1}\left(\alpha_{i} z^{k}\right)^{\ell}\right]
$$


and, inverting the two sums, we have

$$
\begin{aligned}
\sum_{k=0}^{\frac{N_{p}}{b N_{u}}-1} f\left(k b N_{u}+i\right) & =\sum_{\ell=0}^{L_{h}-1} \alpha_{i}^{\ell} \sum_{k=0}^{\frac{N_{p}}{b N_{u}}-1}\left(z^{\ell}\right)^{k} \\
& =\frac{N_{p}}{b N_{u}}+\sum_{\ell=1}^{L_{h}-1} \sum_{k=0}^{\frac{N_{p}}{b N_{u}}-1} \alpha_{i}^{\ell}\left(z^{\ell}\right)^{k}
\end{aligned}
$$

Now, we look at the term $\sum_{k=1}^{\frac{N_{p}}{b N_{u}}-1} f\left(k b N_{u}\right)$ in Equation (36) and observe that, by using the same reasoning, we can write

$$
\sum_{k=1}^{\frac{N_{p}}{b N_{u}}-1} f\left(k b N_{u}\right)=\frac{N_{p}}{b N_{u}}-1+\sum_{\ell=1}^{L_{h}-1} \sum_{k=1}^{\frac{N_{p}}{b N_{u}}-1}\left(z^{\ell}\right)^{k} .
$$

In what follows, we consider two different cases:

(a) The case in which $z^{\ell} \neq 1$. In this case, we observe that $\forall \ell \in\{1,2, \ldots, L-1\}$

$$
\begin{aligned}
\sum_{k=0}^{\frac{N_{p}}{b N_{u}}-1} \alpha_{i}^{\ell}\left(z^{\ell}\right)^{k} & =\alpha_{i}^{\ell} \frac{1-z^{\frac{N_{p}}{b N_{u}}}}{1-z^{\ell}} \\
& =\alpha_{i}^{\ell} \frac{1-e^{-j 2 \pi \ell}}{1-e^{-j 2 \pi \frac{1}{N_{p}} b N_{u} \ell}} \\
& =0 \quad \text { if } z^{\ell} \neq 1 .
\end{aligned}
$$

and

$$
\begin{aligned}
\sum_{k=1}^{\frac{N_{p}}{b N_{u}}-1}\left(z^{\ell}\right)^{k} & =\sum_{k=0}^{\frac{N_{p}}{b N_{u}}-1}\left(z^{\ell}\right)^{k}-1 \\
& =-1 .
\end{aligned}
$$

Therefore, using Equations (38), (39), (40), and (41), Equation (36) becomes

$$
\begin{aligned}
\Gamma(b) & =\sum_{u=1}^{N_{u}} \frac{\sigma_{h}^{(u) 2}}{N_{u}}\left[L_{h}+\frac{N_{p}}{b N_{u}}-1-\left(L_{h}-1\right)+(b-1) \frac{N_{p}}{b N_{u}}\right] \\
& =\sum_{u=1}^{N_{u}} \frac{\sigma_{h}^{(u) 2}}{N_{u}}\left[b \frac{N_{p}}{b N_{u}}\right] \\
& =\sum_{u=1}^{N_{u}} \sigma_{h}^{(u) 2} \frac{N_{p}}{N_{u}^{2}} .
\end{aligned}
$$

(b) The case in which $z^{\ell}=1$. We observe that if there exists an integer $\ell$ in $\left\{1,2, \ldots, L_{h}-1\right\}$ such that $z^{\ell}=1$, then we have

$$
\begin{aligned}
\sum_{k=0}^{\frac{N_{p}}{b N_{u}}-1} \alpha^{\ell}\left(z^{\ell}\right)^{k} & =\sum_{k=0}^{\frac{N_{p}}{b N_{u}}-1} \alpha^{\ell}(1)^{k} \\
& =\alpha^{\ell} \frac{N_{p}}{b N_{u}} \\
& \neq 0 .
\end{aligned}
$$

and

$$
\begin{aligned}
\sum_{k=1}^{\frac{N_{p}}{b N_{u}}-1}\left(z^{\ell}\right)^{k} & =\frac{N_{p}}{b N_{u}}-1 \\
& \neq-1 .
\end{aligned}
$$

Therefore, from the definition of $z=e^{-j 2 \pi \frac{1}{N_{p}} b N_{u}}$, we have that $z^{\ell}=1$ when $\frac{n u}{N_{p}} \in \mathbb{Z}^{+}$. Without loss of generality, we look at the smallest integer in $\mathbb{Z}^{+}$, and we see that

$$
\frac{b N_{u}}{N_{p}} \ell=1 \quad \Leftrightarrow \quad \ell=\frac{N_{p}}{b N_{u}} .
$$

Hence, since $\ell \in\left\{1,2, \ldots, L_{h}-1\right\}$ we have that

$$
\frac{N_{p}}{b N_{u}}<L_{h}
$$

which is equivalent to $b>\frac{N_{p}}{L N_{u}}$. Therefore, when $b>$ $\frac{N_{p}}{L_{h} N_{u}}$, we can have at least one sum of the form

$$
\sum_{k=0}^{\frac{N_{p}}{b N_{u}}-1} \alpha^{\ell}\left(z^{\ell}\right)^{k}>0
$$

(and $\left.\sum_{k=1}^{\frac{N_{p}}{b N_{u}}-1}\left(z^{\ell}\right)^{k}>-1\right)$. From Equations (38), (39), and (42), we can conclude that

$$
\Gamma(b)>\sum_{u=1}^{N_{u}} \sigma_{h}^{(u) 2} \frac{N_{p}}{N_{u}^{2}}, \quad \forall b>\frac{N_{p}}{L_{h} N_{u}} .
$$

To conclude our proof, from the analysis of cases (a) and (b), we can state the following result:

$$
\Gamma_{m}(1)=\cdots=\Gamma_{m}\left(\frac{N_{p}}{L_{h} N_{u}}\right)=\sum_{u=1}^{N_{u}} \sigma_{h}^{(u) 2} \frac{N_{p}}{N_{u}^{2}}
$$

and

$$
\Gamma_{m}(b)>\Gamma_{m}\left(\frac{N_{p}}{L_{h} N_{u}}\right)
$$

for all $b>\frac{N_{p}}{L_{h} N_{u}}$.

Competing interests

The authors declare that they have no competing interests.

\section{Acknowledgements}

The work of the first author has been done while she was with ETIS/ENSEA University of Cergy Pontoise - CNRS Laboratory, Cergy-Pontoise, France. 
Received: 17 February 2014 Accepted: 16 November 2014

Published: 6 December 2014

\section{References}

1. HG Myung, J Lim, DJ Goodman, Single carrier FDMA for uplink wireless transmission. IEEE Vehicular Technol. 1(3), 30-38 (2006)

2. AF Molisch, A Mammela, Taylor D P, Wideband Wireless Digital Communication. (Prentice Hall PTR, Upper Saddle River, NJ, USA, 2001)

3. H Ekstrom, Technical solutions for the 3G Long-Term Evolution. IEEE Commun. Mag. 44(3), 38-45 (2006)

4. HG Myung, in Proceedings of the 15th European Signal Processing Conference. Introduction to Single Carrier FDMA (Poznan, Poland, 2007), pp. 2144-2148

5. PH Moose, A technique for orthogonal frequency division multiplexing frequency offset correction. IEEE Trans. Commun. 42(10), 2908-2914 (1994)

6. H Sari, G Karam, I Jeanclaude, in Proceedings of the 6th Tirrenia International Workshop on Digital Communications. Channel equalization and carrier synchronization in OFDM systems (Tirrenia, Italy, 1993), pp. 191-202

7. Y Zuh, B Letaief, in Proceedings of Global Telecommunication Conference. CFO estimation and compensation in single carrier interleaved FDMA systems (Honolulu, Hawaii, USA, 2009), pp. 1-5

8. A Sohl, A Klein, in Proceedings of the 15th European Signal Processing Conference. Comparison of localized, interleaved, and block-interleaved FDMA in terms of pilot multiplexing and channel estimation (Poznan, Poland, 2007)

9. L Koffman, V Roman, Broadband wireless access solutions based on OFDM access in IEEE 802.16. IEEE Commun. Mag. 40(4), 96-103 (2002)

10. B Aziz, I Fijalkow, M Ariaudo, in Proceedings of Global Telecommunications Conference (GLOBECOM 2011). Tradeoff between frequency diversity and robustness to carrier frequency offset in uplink OFDMA system (Houston, Texas, 2011), pp. 1-5

11. SH Song, GL Chen, KB Letaief, Localized or interleaved? A tradeoff between diversity and CFO interference in multipath channels. IEEE Trans. Wireless Commun. 10(9), 2829-2834 (2011)

12. AM Masucci, I Fijalkow, EV Belmega, in IEEE International Symposium on Personal, Indoor and Mobile Radio Communications (PIMRC). Subcarrier allocation in coded OFDMA uplink systems: Diversity versus CFO (London, United Kingdom, 2013)

13. E Telatar, Capacity of multi-antenna gaussian channels. Eur. Trans. Telecommun. 10, 585-595 (1999)

14. D Tse, P Viswanath, Fundamentals of Wireless Communications. (Cambridge University Press, New York, NY, USA, 2004)

15. WYu, W Rhee, S Boyd, JM Cioffi, Iterative water-filling for gaussian vector multiple-access channels. Inform. Theory, IEEE Trans. 50(1), 145-152 (2004)

16. L Zheng, DNC Tse, Diversity and multiplexing: a fundamental tradeoff in multiple-antenna channels. IEEE Trans. Inform. Theory. 49(5), 1073-1096 (2003)

17. M Godavarti, A Hero, in Proceedings of IEEE International Conference on Acoustic, Speech and Signal Processing. Diversity and degrees of freedom in wireless communications (Orlando, FL, USA, 2002), pp. 2861-2864

18. M-O Pun, M Morelli, CCJ Kuo, Maximum-likelihood synchronization and channel estimation for OFDMA uplink transmissions. IEEE Trans. Commun. 54(4), 726-736 (2006)

\section{Submit your manuscript to a SpringerOpen ${ }^{\odot}$ journal and benefit from:}

- Convenient online submission

- Rigorous peer review

- Immediate publication on acceptance

- Open access: articles freely available online

- High visibility within the field

- Retaining the copyright to your article

Submit your next manuscript at $\boldsymbol{\triangleright}$ springeropen.com 